\title{
Subband/VQ Coding of Color Images with Perceptually Optimal Bit Allocation
}

Robert E. Van Dyke

Sarah A. Rajala

Center for Communications and Signal Processing Department of Electrical and Computer Engineering North Carolina State University

$$
\begin{gathered}
\text { TR-92/9 } \\
\text { July } 1992
\end{gathered}
$$




\title{
Subband/VQ Coding of Color Images
}

\section{With Perceptually Optimal Bit Allocation}

\author{
Robert E. Van Dyck and Sarah A. Rajala \\ Center for Communications and Signal Processing \\ North Carolina State University \\ Raleigh, NC 27695-7914
}

\begin{abstract}
The combination of subband coding and vector quantization can provide a powerful method for compressing color images. The use of properties of the human visual system can increase the performance of such a system and allow one to achieve very high quality reconstructed images at compression ratios exceeding 10:1. In this paper, we design a color subband-vector quantization system, and formulate the bit allocation problem as an optimization problem where the objective function depends on the distortion-rate curves of the quantizers and on a set of perceptual weights. These weights are derived from data provided by experimental measurements $[10,14]$ of the mean detection threshold of the human visual system for color transitions along the luminance, red-green, and blue-yellow directions. Minimization of the objective function constrained by the desired bit rate gives a perceptually optimal bit allocation.

Three subband/VQ cases are examined. In the first two cases (Case 1 and Case 2), the color components of the lowest frequency subband are scalar quantized. Case 1 combines the three color components of each pixel of the higher frequency subbands into a three-dimensional vector, while Case 2 creates four-dimensional vectors from $2 \times 2$ blocks in each subband color component. The third case (Case 3) is the same as Case 2, except that the chrominance components of the lowest frequency subband are also vector quantized with $2 \times 2$ blocks in each component. To obtain the required compression ratio and the high color fidelity required for HDTV applications, the vector quantization is done in two different perceptually uniform color spaces, C.I.E. $L^{*} a^{*} b^{*}$ space and $A C_{1} C_{2}$ space. Results from these color spaces are compared to results obtained in N.T.S.C. $Y I Q$ space.
\end{abstract}




\section{Introduction}

A number of researchers have studied the problem of coding color images with the goal of achieving approximately a 10:1 compression ratio while providing reconstructed images of very high visual quality. Among the many techniques are subband coding and vector quantization. Westerink et al. [24] combined the two methods to code monochrome images. They used the subband coder to filter the image into 16 subbands, and used a sixteen-dimensional vector quantizer where each vector contained the same pixel location from all 16 subbands. Subband/VQ schemes in which the vectors were made from rectangular blocks in each subband have been studied for both monochrome [1] and color images [9]. Combining the three color components of a pixel into a vector and then implementing a vector quantizer has been recently used to design color look-up tables [2]. We have extended this approach to subband coding and have presented some initial results in [21, 22].

In this paper, we compare subband/VQ coders in different color spaces. Two test images, GIRL and DOLL, stored as C.I.E. $X Y Z$ tristimulus values are transformed to N.T.S.C. YIQ, C.I.E. $L^{*} a^{*} b^{*}$, and $A C_{1} C_{2}$ [4] space; the latter two are considered perceptually uniform spaces. Three different vector quantizer cases are simulated. In Case 1, the vectors for each higher frequency subband are created by combining the three color components of the pixels. In Cases 2 and 3 , the components are coded separately with four-dimensional vectors created by taking $2 \times 2$ blocks in each color component. The LBG algorithm [11] with splitting is used to design the codebooks using the minimum mean squared error criterion in the desired color space.

Although subband coding is often suboptimal in a rate-distortion sense [6], by using perceptual information it is possible to overcome this suboptimality and provide high quality reconstructed images. In this work we formulate the bit allocation problem as an optimization problem where the objective function is weighted by the response of the human visual system. A variation of the Marginal Analysis algorithm [7] is used to solve this problem, and sufficient conditions for this to be an optimal solution are stated. A heuristic method is then given to handle the case when the resulting bit allocation is not necessarily optimal.

The objective function uses a set of perceptual weights derived from experimental measurements of the mean detection threshold of the human visual system done by Krishnakumar [10, 14]. The experiments measured the mean detection threshold as a function of spatial frequency, spatial orientation, background luminance, background color, and direction of color transition. The transformation of Krishnakumar's data to the desired color spaces is explained in subsection 4-3, and 
an example is given. The perceptual weights are then derived for each subband color component.

To compare the reconstructed images with the original, we used the $\Delta E$ distortion measure in $L^{*} a^{*} b^{*}$ space. $\Delta E$ is given by

$$
\Delta E=\|\mathbf{x}-\mathbf{y}\|
$$

where $\mathbf{x}$ and $\mathbf{y}$ are the input and output tristimulus vectors in $L^{*} a^{*} b^{*}$ space, and $\|\cdot\|$ is the Euclidean norm. This distortion measure is computed on a pixel by pixel basis and the average over all of the pixels is then compared with the subjective evaluation of the output image. To display the image on a calibrated monitor, the image must be transformed to the RGB space of the display. To achieve the correct colorimetric results, this transformation must compensate for the non-linear transfer functions of the three electron guns. More information on the storage and display of our color images can be found in [20], and the calibration procedure is described in [13].

The outline of this paper is as follows. The next section contains the transformations to the color spaces used in this work. The third section discusses the subband decomposition and the vector quantizer design. The fourth section addresses the perceptually optimal bit allocation problem. The fifth section provides results of computer simulations. The improvement gained by using perceptual weights is shown, and the effects of coding in the different color spaces are discussed. The final section contains the conclusions drawn from this work.

\section{Color Transformations}

The input images used in the computer simulations were recorded as C.I.E. $X Y Z$ tristimulus values. Studies done by MacAdam [12] and others have shown that the human visual system is not equally responsive to the same size color errors in different parts of $X Y Z$ space. One approach to this problem involves the transformation of the color space to another one that has more perceptually uniform color errors. Two such spaces are C.I.E. $L^{*} a^{*} b^{*}$ space and $A C_{1} C_{2}$ space. In this section, the transformations to these color spaces and N.T.S.C. $Y I Q$ space are given. Processing consists of transforming the input image to the desired color space, doing the subband/VQ coding, and then transforming the image back to $X Y Z$ space. 


\section{2-1 Transformation to N.T.S.C. $Y I Q$ Space}

Because of a desire for compatibility with the existing monochrome system, the N.T.S.C. decided to create a color system with one luminance channel, $Y$, and two chrominance channels, $I$ and $Q$. The new color signal had to occupy the same bandwidth as the existing signal, so the chrominance channels were quadrature modulated onto a subcarrier that was then added to the monochrome signal. The chrominance channels are derived from scaled and rotated versions of $(R-Y)$ and $(B-Y)$, where $R$ and $B$ are the red and blue components in N.T.S.C. $R G B$ space. The bandwidth of the chrominance channels had to be reduced to minimize interference with the luminance channel. To reduce the visual degradations as much as possible, a series of experiments were conducted to determine the rotation angle used to create the $I$ and $Q$ axes. An angle of 33 degrees was chosen.

The transformation from $X Y Z$ to $Y I Q$ space takes place in two steps. The first step is the transformation from $X Y Z$ to N.T.S.C. $R G B$ space. The second step is from $R G B$ to $Y I Q$. This is a linear formulation of the N.T.S.C. transformation [17]. The two matrices can be combined to give:

$$
\left[\begin{array}{l}
Y \\
I \\
Q
\end{array}\right]=\left[\begin{array}{rrr}
0.000 & 1.000 & 0.000 \\
1.389 & -0.827 & -0.453 \\
0.938 & -1.195 & 0.233
\end{array}\right]\left[\begin{array}{c}
X \\
Y \\
Z
\end{array}\right]
$$

\section{2-2 Transformation to C.I.E. $L^{*} a^{*} b^{*}$ Space}

$L^{*} a^{*} b^{*}$ space is an almost perceptually uniform color space created by a nonlinear transformation of $X Y Z$ space. The transformation requires the $X Y Z$ coordinates of the desired white point. In our simulations, we used the defined white point of our display; the numerical values are:

$$
\left[\begin{array}{l}
X_{0} \\
Y_{0} \\
Z_{0}
\end{array}\right]=\left[\begin{array}{l}
34.51 \\
37.36 \\
37.16
\end{array}\right]
$$

The transformation from $X Y Z$ to the CIELAB color space $L^{*} a^{*} b^{*}$ is then given by the equations [25]:

$$
\begin{aligned}
& L^{*}=116\left(Y / Y_{0}\right)^{1 / 3}-16 \\
& a^{*}=500\left[\left(X / X_{0}\right)^{1 / 3}-\left(Y / Y_{0}\right)^{1 / 3}\right] \\
& b^{*}=200\left[\left(Y / Y_{0}\right)^{1 / 3}-\left(Z / Z_{0}\right)^{1 / 3}\right]
\end{aligned}
$$


where $X_{0}, Y_{0}$, and $Z_{0}$ are the tristimulus values of white. These formulae are only correct for values of $X / X_{0}, Y / Y_{0}$, and $Z / Z_{0}$ greater than 0.008856 . For lower values of these ratios, $L^{*}=$ 903.29 $\left(Y / Y_{0}\right)$, and $a^{*}$ and $b^{*}$ are also changed [25].

\section{2-3 White Point Mapping of $A C_{1} C_{2}$ Space}

The second perceptually uniform color space that we used is based on a logarithmic transformation of cone space derived by Faugeras [5]. The cone space, $L M S$, is obtained from a linear transformation of $X Y Z$ space [25] using the equation:

$$
\left[\begin{array}{c}
L \\
M \\
S
\end{array}\right]=\left[\begin{array}{rrr}
661.0 & 1260.0 & -112.0 \\
-438.0 & 1620.0 & 123.0 \\
0.708 & 0.0 & 417.0
\end{array}\right]\left[\begin{array}{l}
X \\
Y \\
Z
\end{array}\right]
$$

The transformation to $L M S$ space given by Eq. (4) does not map the desired white point to the origin of the coordinate system. To be consistent with the transformation to $L^{*} a^{*} b^{*}$ space, the transformation was changed so that the monitor's white point is mapped to the origin. Changing the white point is known as white point mapping, and the procedure to calculate the new matrix is now given.

Define the matrix $\mathrm{E}$ to be the transformation matrix in Eq. (4). Let the vector w be the $X Y Z$ tristimulus values and let 1 be the corresponding $L M S$ tristimulus values defined by $1=E w$. It is desired that

$$
\left[\begin{array}{l}
1 \\
1 \\
1
\end{array}\right]=I_{m}=E_{1} \mathbf{w}_{m}
$$

where $\mathbf{E}_{1}$ is the new transformation matrix, and $\mathbf{w}_{m}$ contains the tristimulus values of the monitor's white point with the luminance scaled to unity. The logarithmic processing in Eq. (10) will map the vector $(1,1,1)^{T}$ to $(0,0,0)^{T}$. The white point vector is given by:

$$
\mathbf{w}_{m}=\left[\begin{array}{l}
0.9235 \\
1.0000 \\
0.9945
\end{array}\right]
$$

Now define $E_{1}^{-1}=E^{-1} \Lambda$ where $\Lambda$ is the diagonal matrix of the gains needed to map the white point to the origin. Once $\Lambda$ is found, one can calculate $E_{1}$ and use it instead of $E$ in $E q$. (4). 
Premultiplying both sides of Eq. (5) by $\mathrm{E}_{1}^{-1}$ yields:

This gives:

$$
\mathbf{w}_{m}=\mathbf{E}_{1}^{-1}\left[\begin{array}{l}
1 \\
1 \\
1
\end{array}\right]=\mathbf{E}^{-1} \boldsymbol{\Lambda}\left[\begin{array}{l}
1 \\
1 \\
1
\end{array}\right]
$$

Finally, the new matrix is: $\mathbf{E}_{1}=\Lambda^{-1} \mathbf{E}$.

$$
\Lambda\left[\begin{array}{l}
1 \\
1 \\
1
\end{array}\right]=\mathbf{E} \mathbf{w}_{m}=\left[\begin{array}{c}
1759.32 \\
1337.67 \\
415.57
\end{array}\right]
$$

\section{2-4 Transformation to $A C_{1} C_{2}$ Space}

The transformation from $X Y Z$ to $A C_{1} C_{2}$ space occurs in three steps. The first step is a linear transformation from $X Y Z$ space to cone space using matrix $\mathrm{E}_{1}$ derived in the last section. This is followed by a logarithmic mapping and another linear transformation. The processing is as follows:

$$
\left[\begin{array}{c}
L \\
M \\
S
\end{array}\right]=\left[\begin{array}{rrr}
0.37512 & 0.71618 & -0.06366 \\
-0.32743 & 1.21106 & 0.09195 \\
0.00170 & 0.00000 & 1.00344
\end{array}\right]\left[\begin{array}{l}
X \\
Y \\
Z
\end{array}\right]
$$

$$
\left[\begin{array}{l}
A \\
C_{1} \\
C_{2}
\end{array}\right]=\left[\begin{array}{rrr}
13.8310 & 8.3394 & 0.4294 \\
64.0000 & -64.0000 & 0.0000 \\
10.0000 & 0.0000 & -10.0000
\end{array}\right]\left[\begin{array}{l}
l \\
m \\
s
\end{array}\right]
$$

The last transformation is the achromatic-chromatic separation. It creates the $A$ channel containing the luminance information and two chrominance channels. $C_{1}$ contains red-green information and $C_{2}$ contains blue-yellow information. Frequency selective filtering can be applied to this color space to create the color space $A^{*} C_{1}^{*} C_{2}^{*}$. Since the subband coding is also frequency selective, this filtering was not done. The human visual system's frequency dependence will be considered in the bit allocation algorithm. 


\section{Subband/VQ Design}

The subband simulation first filters the color image into four subbands using the 32 tap QMF filter D from Johnston [8]. The lowpass filtered subband is further filtered into three more subbands to yield a total of seven. The filtering is done in the time domain with constant extension at the boundaries to minimize edge effects. The color images were compressed by creating three such systems in parallel, where each system processes one of the three color components. By transforming to the desired color space before the transmitter filterbank and transforming back after the receiver filterbank, the processing can be done in any color space. A different set of codebooks is used for each color space.

Because of the large number of levels that are needed for the lowest frequency subband, each of its components are scalar quantized in Cases 1 and 2. The other six subbands are all vector quantized. Simulations of Case 3 are run only at high compression ratios in order to reduce the required number of levels for the lowest frequency chrominance components. The LBG algorithm with splitting [11] is used to design the codebooks in all cases. A full search VQ is used since the maximum number of levels needed for each subband is not large. The use of splitting to determine the initial codevectors for the LBG algorithm requires that codebooks for all rates smaller than a given rate be found first. By doing this, one can obtain a distortion-rate curve for the particular vector quantizer. These curves will be used for the bit allocation process.

To obtain the training sequence, four training images are each filtered into seven subbands. For each subband color component, a single subimage is created from the subimages of the four training images. This can be contrasted with the simulations performed in [22], where only a single image was used as the training sequence. For Case 1, the three color components of the pixels in each higher frequency subband are combined into vectors and these vectors become the training sequence for that subband. Nine codebooks must be designed, three for the components of the lowest subband, and one codebook for each of the other six subbands. For Case 2, each block of four pixels in a color component of a higher frequency subband is considered as a vector. The vectors for that component and subband are then used as the training sequence. Now, a total of 21 codebooks must be designed, one for each component of the seven subbands. These procedures were repeated for each desired color space. 


\section{Perceptually Optimal Bit Allocation}

\section{4-1 Introduction}

As in all subband coding schemes, one must determine how to allocate bits among the subbands. In color subband coding, one faces the additional problem of allocating bits among the color components of each subband if the components are treated separately. A number of algorithms have been proposed to solve this problem. They can generally be classified into two separate categories. The first method uses a model of the optimal scalar quantizer and the variances of the subband components to provide a mathematically suboptimal, yet useful, bit allocation. Because the problem is solved by using a continuous Lagrange multiplier method, the results are not constrained to non-negative integers. The non-negativity constraint can, however, be added [15], but the results are still real numbers. These can be rounded to the nearest integer and adjusted, if necessary, to yield the required bit allocation.

The second method was first given by Fox [7] using the generalized Lagrange multiplier method derived by Everett [3]. This method, known as Marginal Analysis, was originally used to solve military allocation problems, and was used by Trushkin $[18,19]$ for bit allocation of vector quantizers. Shoham and Gersho [16] proposed an extension of this method for arbitrary quantizers where the distortion-rate curves were neither convex nor monotonic. Westerink et al. [23] generalized Trushkin's work to allow non-integer bit allocations, and then they used their version for the monochrome subband case. Because Marginal Analysis uses the actual distortion-rate curves for the quantizers, any type of quantizer can be implemented. Scalar and vector quantizers can be used for different subband components in the same system. This is important since it is desirable to scalar quantize each color component of the lowest subband because of the large required number of levels, and vector quantize the higher subbands to achieve a greater compression ratio.

The latter method will be described in the following subsections. It has been used to obtain the bit allocation when the higher frequency subbands were vector quantized. We propose an extension of the method for subband coding of color images by adding weights to the cost functions based on properties of the human visual system. This generalization is important since the human visual system not only has different responses for the color components, but these responses are also frequency dependent. The new bit allocation scheme will attempt to provide the perceptually optimal solution. 


\section{4-2 Marginal Analysis Method}

In this section, we show how the method of Marginal Analysis can be used to provide an optimal solution to the bit allocation problem. The discussion of this method will illustrate the case where each component of the lowest frequency subband is scalar quantized and the other six subbands are vector quantized by combining the three color components of each pixel into a three-dimensional vector (Case 1). The scalar case and the vector quantizer cases where the vectors are created from blocks of pixels in each color component (Cases 2 and 3 ) are treated similarly.

The problem can be formulated as follows:

$$
\min _{\mathbf{b} \in S} D(\mathbf{b})
$$

subject to

$$
C(\mathbf{b}) \leq B
$$

where $D(\mathbf{b})$ is the distortion function, $B$ is the total bit rate, and $C(\mathbf{b})$ is the number of bits required by strategy b. The set $S$ is the set of all possible bit allocations among the subbands.

The bit allocation problem reduces to the cell problem [3] because the mean squared errors for each vector quantizer are summed to obtain the total distortion. The optimization problem is now:

$$
\min _{b_{i} \in S_{i}} \sum_{c=1}^{3} D_{1, c}\left(b_{1, c}\right) / w_{1, c}+\sum_{i=2}^{7} D_{i}\left(b_{i}\right) / w_{i}
$$

subject to

$$
\sum_{c=1}^{3} C_{1, c}\left(b_{1, c}\right)+\sum_{i=2}^{7} C_{i}\left(b_{i}\right) \leq B
$$

$D_{i}\left(b_{i}\right)$ is the distortion-rate curve for subband $i$. The second subscript for the first subband is used to designate the three color components, since they are scalar quantized. The functions $C_{i}\left(b_{i}\right)=c_{i} b_{i}$, where the cost factors $c_{i}$ are needed because of the different sizes of the subbands. The cost functions are given in Tables 1 and 2 for Cases 1, 2, and 3. The $w_{i}$ are perceptual weighting factors that are determined by the visual importance of the subband and color component. They will be discussed in more detail later. Since there are seven subbands and the lowest has three components, $\mathbf{b}$ is a nine-dimensional vector, $\mathbf{b}=\left(b_{1,1} b_{1,2} b_{1,3} b_{2} \cdots b_{7}\right)^{T}$. The components of the vector can be relabeled to give $\mathbf{b}=\left(b_{1} \cdots b_{9}\right)^{T}$.

Figure 1 shows the mean squared error versus bit rate for subbands 112, 121, and 122 in $L^{*} a^{*} b^{*}$ space. One can see that these functions are convex. The definition of convex for functions 


\begin{tabular}{|c||c|c|c|}
\hline Subband & Lum & R-G & B-Y \\
\hline 111 & $1 / 16$ & $1 / 16$ & $1 / 16$ \\
\hline 112 & \multicolumn{3}{|c|}{$1 / 16$} \\
121 & \multicolumn{3}{|c|}{$1 / 16$} \\
122 & \multicolumn{3}{|c|}{$1 / 16$} \\
\hline 12 & \multicolumn{3}{|c|}{$1 / 4$} \\
21 & & $1 / 4$ \\
22 & & $1 / 4$ \\
\hline
\end{tabular}

Table 1: Subband Costs - Case 1. The costs depend on the size of the subbands. For the scalar quantizers these are the costs of adding one bit/pixel. For the vector quantizers these are the costs of adding one bit/vector. Lum refers to the luminance component, R-G refers to the red-green component, and B-Y refers to the blue-yellow component. The vectors include the three color components so there is only one weight per subband.

\begin{tabular}{|c||c|c|c||c|c|c|}
\hline \multicolumn{1}{|c||}{} & \multicolumn{3}{c||}{ Case 2 } & \multicolumn{3}{c|}{ Case 3 } \\
Subband & Lum & R-G & B-Y & Lum & R-G & B-Y \\
\hline 111 & $1 / 16$ & $1 / 16$ & $1 / 16$ & $1 / 16$ & $1 / 64$ & $1 / 64$ \\
\hline 112 & $1 / 64$ & $1 / 64$ & $1 / 64$ & $1 / 64$ & $1 / 64$ & $1 / 64$ \\
121 & $1 / 64$ & $1 / 64$ & $1 / 64$ & $1 / 64$ & $1 / 64$ & $1 / 64$ \\
122 & $1 / 64$ & $1 / 64$ & $1 / 64$ & $1 / 64$ & $1 / 64$ & $1 / 64$ \\
\hline 12 & $1 / 16$ & $1 / 16$ & $1 / 16$ & $1 / 16$ & $1 / 16$ & $1 / 16$ \\
21 & $1 / 16$ & $1 / 16$ & $1 / 16$ & $1 / 16$ & $1 / 16$ & $1 / 16$ \\
22 & $1 / 16$ & $1 / 16$ & $1 / 16$ & $1 / 16$ & $1 / 16$ & $1 / 16$ \\
\hline
\end{tabular}

Table 2: Subband Costs - Cases 2 and 3. The costs depend on the size of the subbands. For the scalar quantizers these are the costs of adding one bit/pixel. For the vector quantizers these are the costs of adding one bit/vector. Lum refers to the luminance component, $R-G$ refers to the red-green component, and B-Y refers to the blue-yellow component.

defined only on the integers is that the first differences are all negative and increasing as the bit rate increases. When all of the distortion-rate curves are convex, the problem of finding the optimal bit allocation is considerably simplified. The Marginal Analysis algorithm will give the optimal solution.

\section{4-2.1 Marginal Analysis Algorithm}

The allocation algorithm is iterative, and is given as follows:

1. Initially set $\mathbf{b}^{0}=(0 \cdots 0)^{T}$. Set $k=1$.

2. $\mathbf{b}^{k}=\mathbf{b}^{k-1}+\mathbf{e}_{i}$, where $\mathbf{e}_{i}$ is the $i$ th unit vector and $i$ is the index which has the maximum

$$
\left[D_{j}\left(b_{j}^{k-1}\right)-D_{j}\left(b_{j}^{k-1}+1\right)\right] /\left(w_{j} c_{j}\right)
$$

3. If $C\left(\mathbf{b}^{k}\right)>B$ then stop, else $k=k+1$ and go to 2 . 


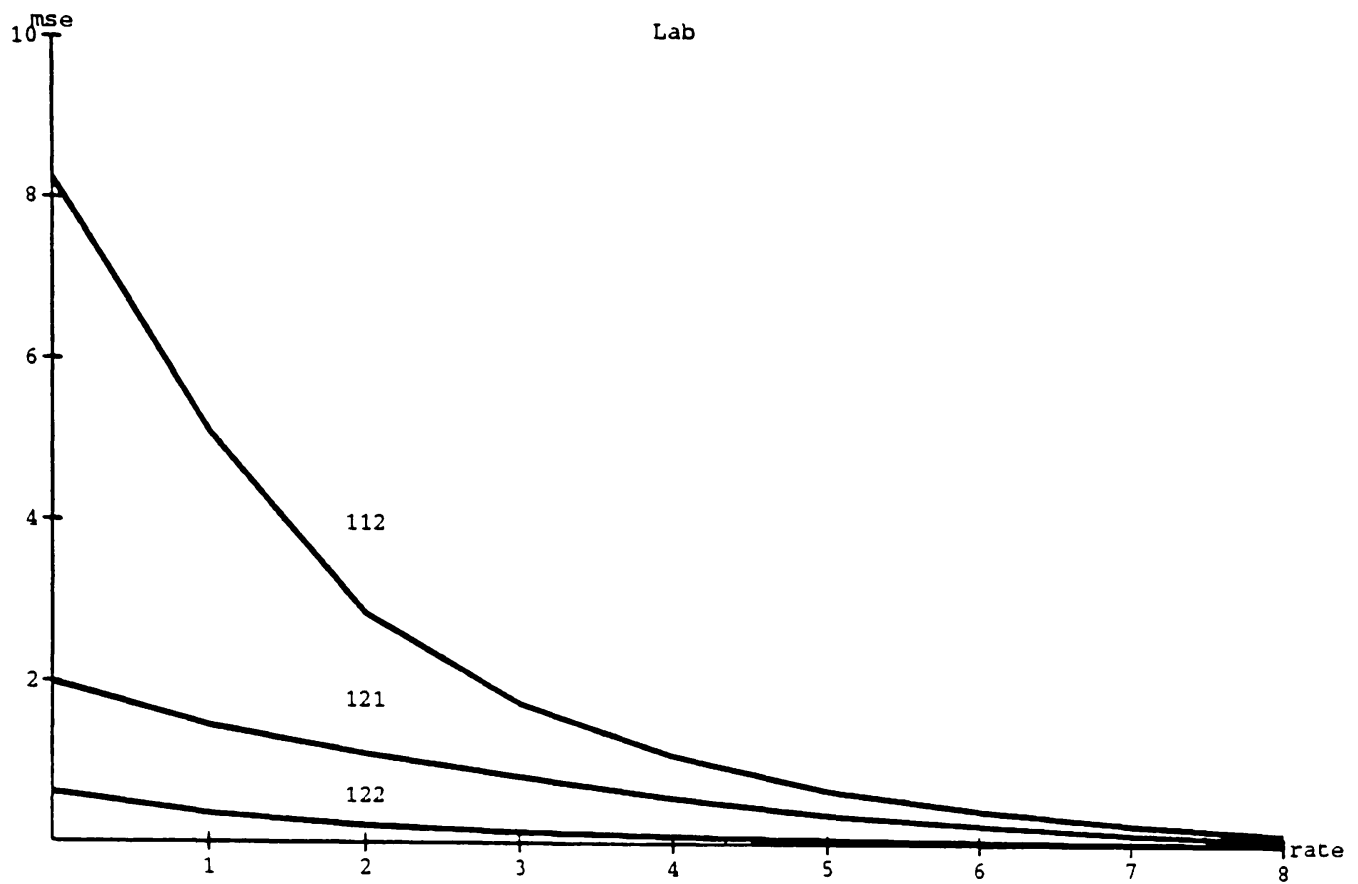

Figure 1: Distortion-Rate Curves of Subbands 112, 121, and 122 in $L^{*} a^{*} b^{*}$ Space.

When the algorithm terminates, one may have to adjust the last couple of allocations so the total bit rate matches $B$ and does not exceed it. If all of the subbands where the same size, this would not be necessary.

\section{4-2.2 Quasiconvexity Condition}

The Marginal Analysis algorithm is guaranteed to provide the optimal solution only if all of the rate distortion curves are convex. Unfortunately, this may not be true, especially for Case 2 and Case 3. The LBG algorithm is only guaranteed to converge to a local minimum. The change in distortion between bit rate $i-1$ and $i$ is not always greater than the change between bit rate $i$ and $i+1$. This non-convexity most often occurs for the higher frequency subbands where the increase is from a bit rate of zero bits/pixel to a bit rate of one bit/pixel.

The quasiconvexity condition is a sufficient condition for the bit allocation to be optimal [19]. This condition is:

$$
s_{i}\left(\mathbf{b}^{*}\right) \leq S_{j}\left(\mathbf{b}^{*}\right) ; \quad i, j=1, \cdots, 9 ; i \neq j
$$

where 


$$
\begin{aligned}
s_{i}\left(\mathbf{b}^{*}\right) & =\max _{b_{i}^{*} \leq b_{i}<B}\left(D_{i}\left(b_{i}\right)-D_{i}\left(b_{i}+1\right) /\left(w_{i} c_{i}\right)\right), \\
S_{j}\left(\mathbf{b}^{*}\right) & =\min _{0 \leq b_{j}<b_{j}}\left(D_{j}\left(b_{j}\right)-D_{j}\left(b_{j}+1\right) /\left(w_{j} c_{j}\right)\right),
\end{aligned}
$$

and $\mathbf{b}^{*}=\left(b_{1}^{*} \cdots b_{9}^{*}\right)^{T}$ is the bit allocation from the Marginal Analysis algorithm.

The bit allocation algorithm first uses Marginal Analysis to find an initial allocation. If the quasiconvexity condition is satisfied, the allocation is optimal and the procedure is finished. If not, the subbands or subband components that cause the nonconvexity are determined and the locations on the distortion-rate curves where convexity is lost are found. This information is provided by the bit allocation program. Since the program lists the order that bits are allocated to the various subbands, a procedure of pair-wise changes is used. The total distortion is first calculated and then a pair-wise change is made in the bit allocation. The new distortion is calculated and compared to the previous one. If the distortion is lower, the new allocation is kept.

\section{4-3 Mean Detection Threshold}

Initially, the perceptual weights, $w_{j}$, were all set to unity. However, the human visual system is both color and frequency dependent. Subjective experiments have shown [10] that even in a perceptually uniform color space such as $L^{*} a^{*} b^{*}$ space, the mean detection threshold is a function of spatial frequency, orientation, luminance, background color, and direction of the color transition. Using this research, we have derived sets of weighting factors. These sets give a numerical weight for each subband color component, and are based on a specified viewing distance from the image. In the following, a description of how the mean detection threshold data was measured and how it can be transformed into the different color spaces is described.

\section{4-3.1 Experimental Measurements}

The experiments [10] measured the smallest change in a color necessary for a human observer to notice that change. Sinusoidal variations in color at different spatial frequencies were displayed on a monitor, and the amplitude of each variation was increased until the pattern was visible or an upper limit was reached. The pattern was then made clearly visible and the amplitude was decreased until the pattern was not visible or a lower limit was reached. The average of a number

of test subjects gives the mean detection threshold. The details of these experiments can be found in $[10,14]$. 
The initial data was measured in $x y Y$ space where $x$ and $y$ are the C.I.E. chromaticity coordinates and $Y$ is the luminance. Four representative chromaticities were chosen, one each in the white, red, green, and blue regions of the monitor's gamut. The chromaticity coordinates for these points are given in Table 3. About these points, the mean detection thresholds were measured for transitions along the luminance, red-green, and blue-yellow directions for six different spatial frequencies and for $Y$ tristimulus values of 5,10 , and $20 \mathrm{~cd} / \mathrm{m}^{2}$. Figure 2 shows the redgreen and blue-yellow directions on the $x y$ chromaticity diagram for transitions about the white point, $(x, y)=(0.33,0.35)$. Included on the plot is the spectral locus and the monitor's gamut for $Y=5 \mathrm{~cd} / \mathrm{m}^{2}$.

\begin{tabular}{|l|c|c|}
\hline Hue & \multicolumn{2}{|c|}{ Chromaticity Coordinates } \\
& $x$ & $y$ \\
\hline White & 0.33 & 0.35 \\
Red & 0.42 & 0.39 \\
Green & 0.34 & 0.46 \\
Blue & 0.29 & 0.29 \\
\hline
\end{tabular}

Table 3: Representative Chromaticities. These are the four background colors used in the visual sensitivity experiments.

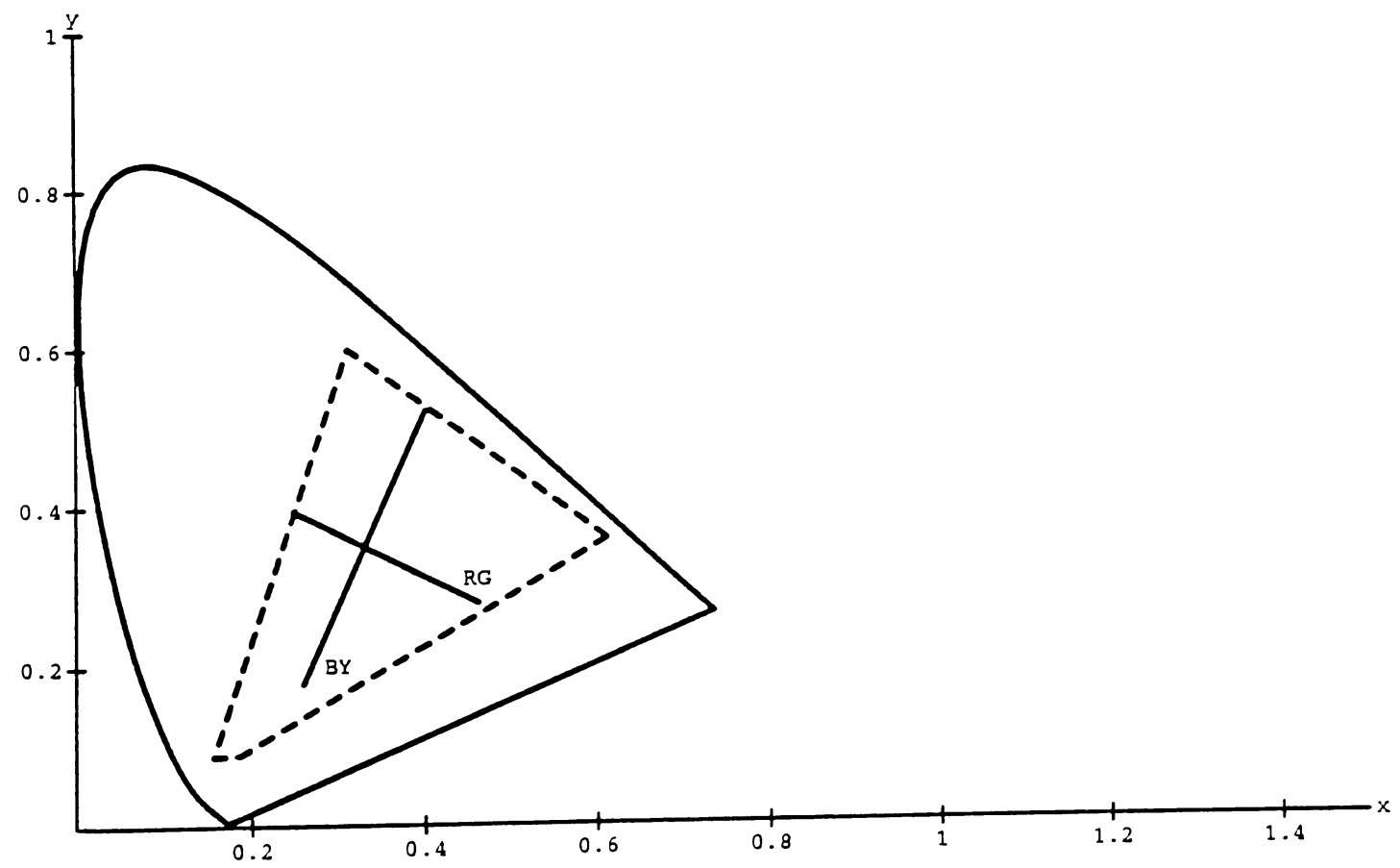

Figure 2: Directions of Variation about the White Point. The white point's chromaticity is $(0.33,0.35)$. The monitor's gamut for $Y=5 \mathrm{~cd} / \mathrm{m}^{2}$ is also plotted. 
The equations for the red-green and blue-yellow directions are given parametrically as:

$$
\begin{aligned}
& x=x_{0} \pm \Delta x \times t \\
& y=y_{0} \pm \Delta y \times t
\end{aligned}
$$

where $t$ is the mean detection threshold, and the point $\left(x_{0}, y_{0}\right)$ is one of the four representative chromaticities. $\Delta x$ and $\Delta y$ are the changes in the $x$ and $y$ chromaticity coordinates, and they are different for the red-green and blue-yellow directions. Table 4 gives the values of $\Delta x$ and $\Delta y$ for all three directions of color transition.

\begin{tabular}{|l|c|c|c|}
\hline Direction & $\Delta Y$ & $\Delta x$ & $\Delta y$ \\
\hline Luminance & 0.0124 & 0.0 & 0.0 \\
Red-Green & 0.0 & 0.000327 & -0.000179 \\
Blue-Yellow & 0.0 & 0.000141 & 0.000345 \\
\hline
\end{tabular}

Table 4: Amounts of Change for Each Direction.

For a constant luminance, the $x y$ contrast is given by $\Delta x y=\left((\Delta x)^{2}+(\Delta y)^{2}\right)^{1 / 2}$. The data was measured in contrast units corresponding to an absolute difference of $\Delta x y=0.000745$. Since the variations in color were sinusoidal about the center point, the contrast is equivalent to twice the variation from the center to either end point. Therefore,

$$
2 \times\left((0.000327)^{2}+(0.000179)^{2}\right)^{1 / 2}=2 \times\left((0.000141)^{2}+(0.000345)^{2}\right)^{1 / 2}=0.000745
$$

For the luminance direction, the chromaticity coordinates remain unchanged and only the luminance value is varied.

\section{4-3.2 Transformation to $X Y Z$ Space}

To convert the mean detection threshold data to the color spaces $Y I Q, L^{*} a^{*} b^{*}$, and $A C_{1} C_{2}$, the data must first be transformed from $x y Y$ space to $X Y Z$ space. The procedure is different for the luminance and chrominance changes, so each one will be discussed. For both the red-green and blue-yellow directions, Eq. (19) provides two chromaticity coordinates for each mean detection threshold value. Call these two points $\left(x_{1}, y_{1}, z_{1}\right)$ and $\left(x_{2}, y_{2}, z_{2}\right)$, where $z_{i}=1-x_{i}-y_{i}$ for $i=1,2$. These two points are then converted to $X Y Z$ space for the given value of $Y$. That is,

$$
Y_{i}=Y \quad X_{i}=x_{i} Y / y_{i} \quad Z_{i}=z_{i} Y / y_{i}
$$

for $i=1,2$. 
For the luminance direction, each mean detection threshold value provides two $X Y Z$ tristimulus values. The equations are:

$$
\begin{gathered}
X_{i}=X_{0} \pm \Delta Y \times t \\
Y_{i}=Y_{0} \pm \Delta Y \times t \\
Z_{i}=Z_{0} \pm \Delta Y \times t
\end{gathered}
$$

where $\Delta Y$ is given in Table $4, t$ is the mean detection threshold, and $i=1,2$. The tristimulus vector $\left(\begin{array}{lll}X_{0} & Y_{0} & Z_{0}\end{array}\right)^{T}$ contains the tristimulus values of the center point at the desired luminance value, $Y$. It is computed in the same way as Eq. (21).

Once the data has been converted to $X Y Z$ space, the mean detection threshold in this color space can be computed. It is the Euclidean norm of the difference of the two tristimulus vectors. The value is given by:

$$
t_{X Y Z}=\left\|\mathbf{T}_{1}-\mathbf{T}_{2}\right\|
$$

where $\mathrm{T}_{1}=\left(\begin{array}{lll}X_{1} & Y_{1} & Z_{1}\end{array}\right)^{T}$ and $\mathrm{T}_{2}=\left(\begin{array}{lll}X_{2} & Y_{2} & Z_{2}\end{array}\right)^{T}$. The mean detection thresholds in the color spaces $L^{*} a^{*} b^{*}, A C_{1} C_{2}$, and $Y I Q$ are the Euclidean norms of the differences of the corresponding vectors obtained by transforming the two $X Y Z$ tristimulus vectors to the desired color space.

\section{4-3.3 Transformation to $L^{*} a^{*} b^{*}$ Space}

The transformation from $X Y Z$ space to $L^{*} a^{*} b^{*}$ is defined in terms of the tristimulus values of a white point. Using the monitor's white point, the red-green and blue-yellow directions in $L^{*} a^{*} b^{*}$ space are shown in Figure 3. The data was measured with $Y=5 \mathrm{~cd} / \mathrm{m}^{2}$, and this maps to $L^{*}=43.34$. The red-green direction is essentially along the $a^{*}$ axis as is desired. However, the blue-yellow direction is not along the $b^{*}$ axis. Since the transformation uses a white point that is not the same as the white point about which the data was measured, there is a slight offset as seen in the plot.

\section{4-3.4 Transformation to $A C_{1} C_{2}$ Space}

The transformation from $X Y Z$ to $A C_{1} C_{2}$ space consists of three parts as was discussed previously. The first part is a linear transformation to the cone space, $L M S$. The mapping used by Faugeras [5] uses the transformation from [25]. He uses a white point mapping such that either standard illuminant $\mathrm{C}$ or illuminant $D_{65}$ with unity luminance maps to the origin. 


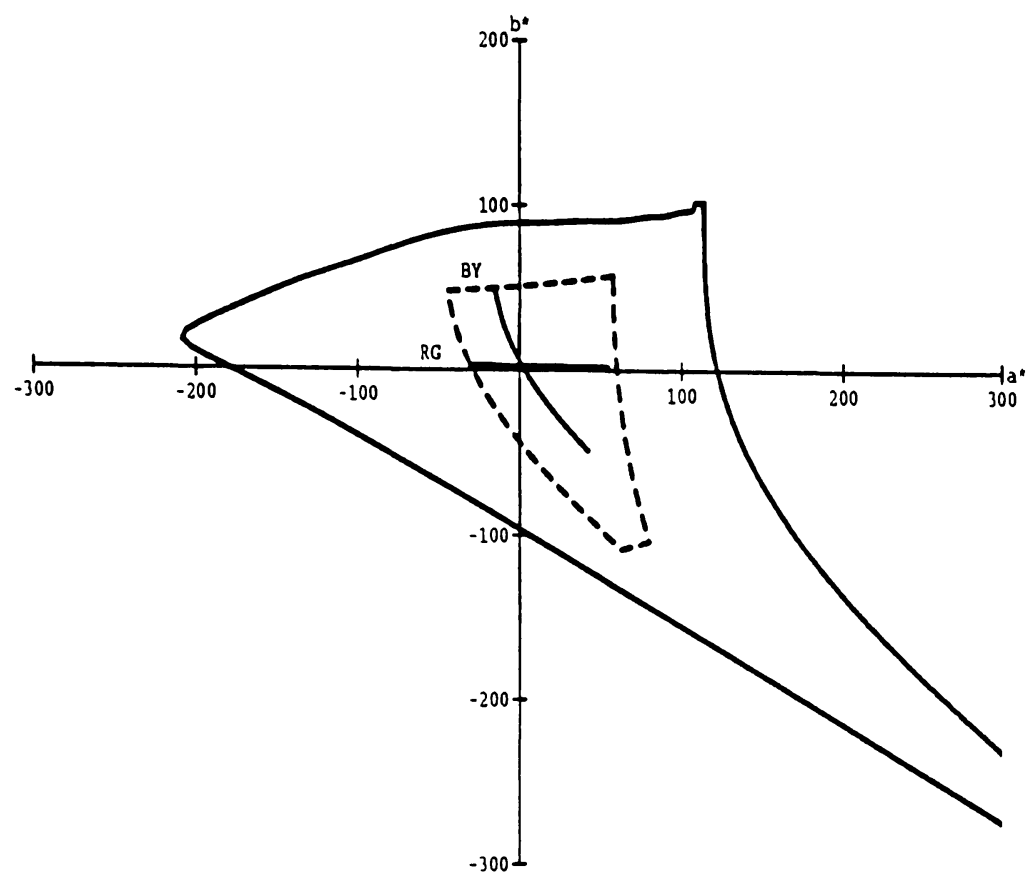

Figure 3: Directions of Variation in $L^{*} a^{*} b^{*}$ Space. White Point Chromaticity is $(0.3165,0.3426)$. $Y=5 \mathrm{~cd} / \mathrm{m}^{2}$.

It is simple enough to derive a transformation to $A C_{1} C_{2}$ space such that any desired white point maps to the origin. While standard illuminant $C$ is used as the white point in the N.T.S.C. $X Y Z$ to $Y I Q$ transformation, the use of the monitor's white point would be more consistent with its use in the transformation to $L^{*} a^{*} b^{*}$ space. This transformation was implemented, and the redgreen and blue-yellow directions are shown in Figure 4. Again, one notices that there is an offset between the center of the color transitions and the origin. More importantly, these two directions of color transition are almost orthogonal and are very close to the axes.

\section{4-3.5 Transformation to $Y I^{*} Q^{*}$ Space}

The transformation to $Y I Q$ space is a linear one, so the red-green and blue-yellow lines map to straight lines. The red-green line lies mostly in the in-phase, $I$, direction, and the blue-yellow line lies mostly in the quadrature, $Q$, direction. The angles that these lines have been rotated through, relative to the $I$ axis, depend on the white point about which the lines were constructed. For the white point of $(0.33,0.35)$, the angles for the red-green and blue-yellow directions are 34.47 and -73.26 degrees. Notice that these lines are not orthogonal to each other. Using standard illuminant $\mathrm{C}$ as the center changes these rotation angles by less than $2^{\circ}$. 


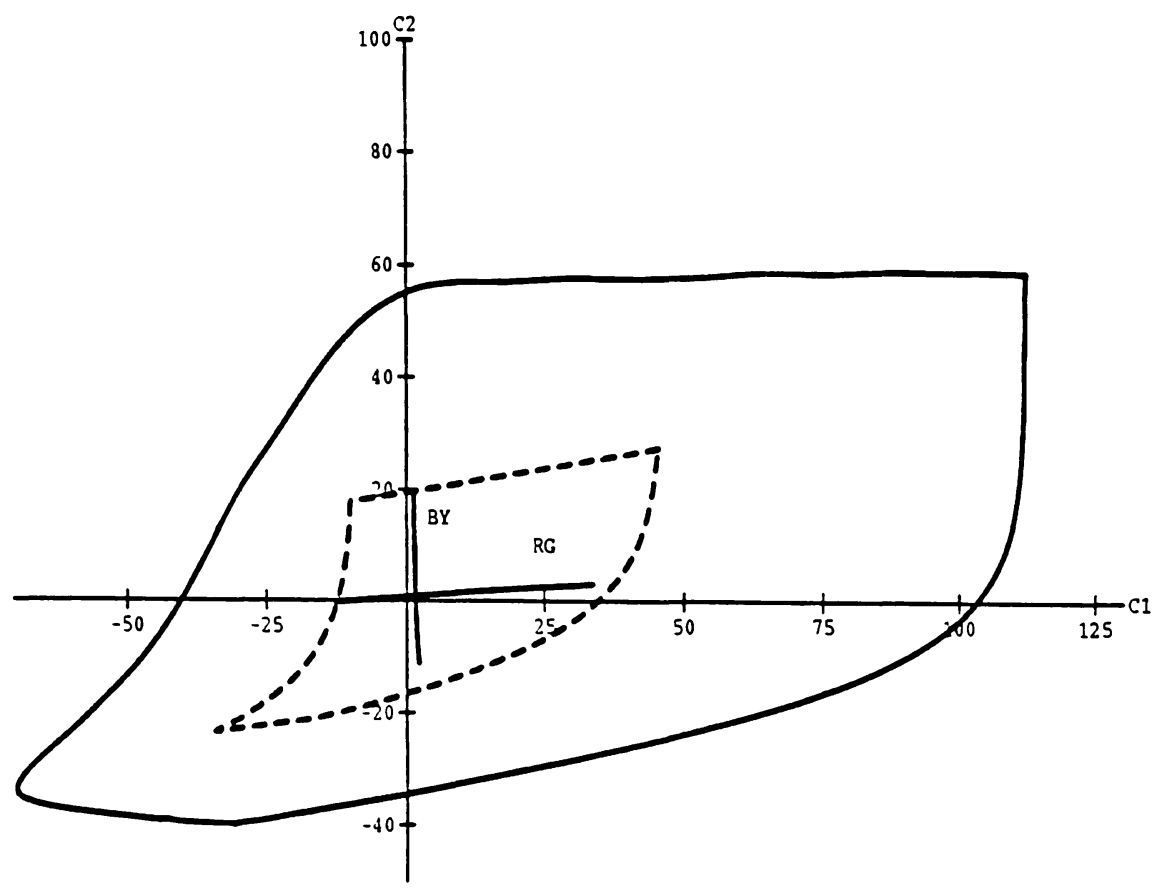

Figure 4: Directions of Variation in $A C_{1} C_{2}$ Space. White Point Chromaticity is $(0.3165,0.3426)$. $Y=5 \mathrm{~cd} / \mathrm{m}^{2}$.

In order to more optimally match the data in the visual experiment, a variation of $Y I Q$ space where the directions of the chrominance axes are closer to the directions used in the experiment would be preferable. This makes the perceptual weights more meaningful, and it also allows for a more accurate comparison with $L^{*} a^{*} b^{*}$ and $A C_{1} C_{2}$ space. Therefore, a new color space, designated $Y I^{*} Q^{*}$ was derived; the details are now given.

The new color space is based on the N.T.S.C. phosphors and the white point of our color monitor. First, a transformation to $X Y Z$ space from a new $R G B$ space is derived. This $R G B$ space is created by determining the gains on each of the red, green, and blue channels so that an $R G B$ vector of $\left(\begin{array}{lll}1 & 1 & 1\end{array}\right)^{T}$ maps to an $X Y Z$ vector equal to the monitor's white point with the luminance scaled to unity. The transformation is:

$$
\left[\begin{array}{l}
X \\
Y \\
Z
\end{array}\right]=\left[\begin{array}{lll}
0.5734 & 0.1842 & 0.1659 \\
0.2824 & 0.6228 & 0.0948 \\
0.0000 & 0.0702 & 0.9243
\end{array}\right]\left[\begin{array}{l}
R \\
G \\
B
\end{array}\right] .
$$

The $I^{*}$ and $Q^{*}$ channels are given by:

$$
\begin{aligned}
I^{*} & =(R-Y) / 1.14 \\
Q^{*} & =(B-Y) / 2.03
\end{aligned}
$$


Notice that so far, the only differences from N.T.S.C. $Y I Q$ space is that a different white point is used and there is no rotation of the $(R-Y) / 1.14$ and $(B-Y) / 2.03$ axes.

Since the $Y$ in $X Y Z$ space is the same as the $Y$ in $Y I^{*} Q^{*}$ space, one can combine Eqs. (24) and (25) to get:

$$
\left[\begin{array}{l}
Y \\
I^{*} \\
Q^{*}
\end{array}\right]=\left[\begin{array}{rrr}
0.2824 & 0.6228 & 0.0948 \\
0.6295 & -0.5463 & -0.0832 \\
-0.1391 & -0.3068 & 0.4459
\end{array}\right]\left[\begin{array}{l}
R \\
G \\
B
\end{array}\right]
$$

Replacing the $(R G B)^{T}$ vector in Eq. (26) with the inverse of Eq. (24) gives the desired result:

$$
\left[\begin{array}{l}
Y \\
I^{*} \\
Q^{*}
\end{array}\right]=\left[\begin{array}{rrr}
0.0000 & 1.0000 & 0.0000 \\
1.7733 & -1.3715 & -0.2676 \\
0.0347 & -0.5630 & 0.5339
\end{array}\right]\left[\begin{array}{l}
X \\
Y \\
Z
\end{array}\right] .
$$

The red-green and blue-yellow directions in this color space are shown in Figure 5. The red-green line is now along the $I^{*}$ axis. The blue-yellow line is at an angle of approximately 14.4 degrees from the $Q^{*}$ axis.

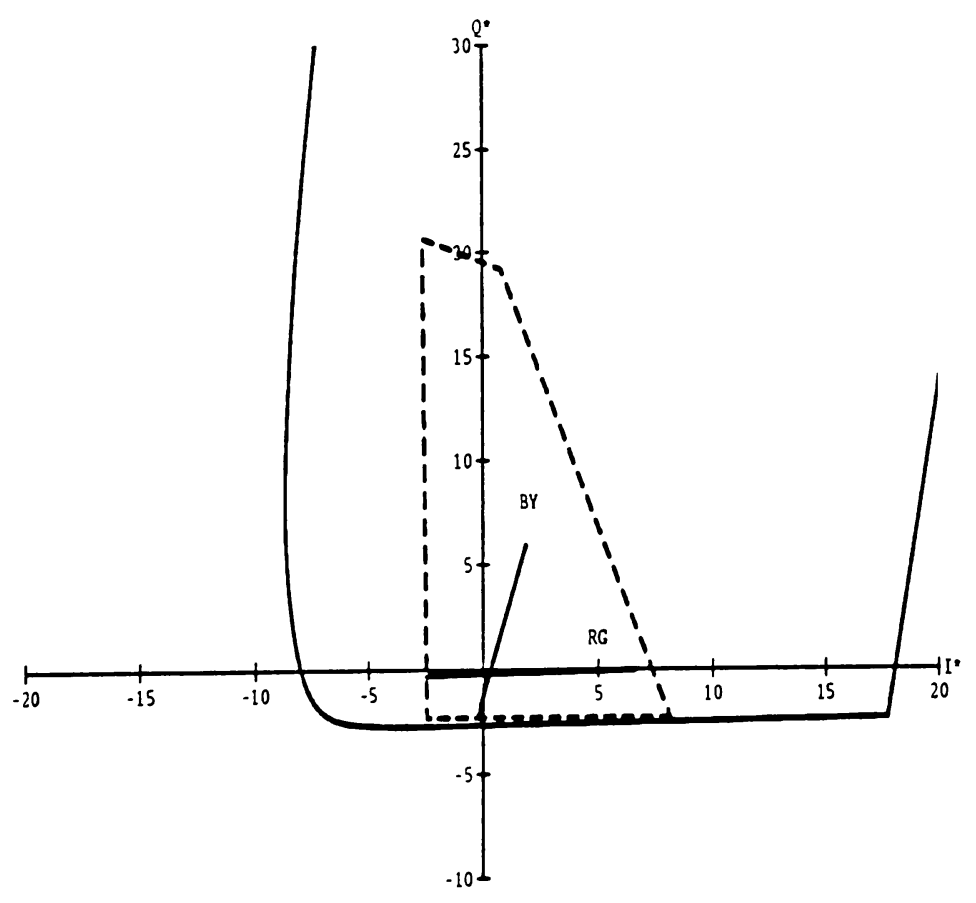

Figure 5: Directions of Variation in $Y I^{*} Q^{*}$ Space. White Point Chromaticity is $(0.3165,0.3426)$. $Y=5 \mathrm{~cd} / \mathrm{m}^{2}$. 


\section{4-3.6 Example Transformation of Mean Threshold Data}

The following example will illustrate how the mean threshold data was transformed from $x y Y$ to $L^{*} a^{*} b^{*}$ space using the monitor's white point in the transformation. Table 5 contains contains the mean threshold data as originally recorded in $x y Y$ space. The entries with a single asterisk by them indicate that at least one of the experimental subjects was unable to detect the pattern. A double asterisk indicates that no subjects were able to detect the pattern; the value of 150 was chosen. This value was selected since it is higher than any of the measured data, but low enough to allow the possibility of a non-zero bit allocation.

\begin{tabular}{|c|c|c|c|c|c|c|c|}
\hline \multirow{2}{*}{$\begin{array}{l}\text { Spatial } \\
\text { Dir. }\end{array}$} & \multirow{2}{*}{$\begin{array}{l}\text { Color } \\
\text { Dir. }\end{array}$} & \multicolumn{6}{|c|}{ Spatial Frequency cycles/deg } \\
\hline & & 0.5 & 1.0 & 2.0 & 5.0 & 10.0 & 20.0 \\
\hline \multirow[t]{3}{*}{ Hor. } & Lum & 8.167 & 6.750 & 6.333 & 7.250 & 13.500 & 65.083 \\
\hline & $\mathrm{R}-\mathrm{G}$ & 4.667 & 4.750 & 4.750 & 7.167 & 17.417 & 77.417 \\
\hline & $\mathrm{B}-\mathrm{Y}$ & 11.667 & 6.000 & 6.833 & 32.667 & 70.167 & $* * 150.000$ \\
\hline \multirow[t]{3}{*}{ Vert. } & Lum & 7.000 & 6.833 & 6.250 & 6.833 & 22.500 & *77.800 \\
\hline & $\overline{R-G}$ & 5.083 & 5.583 & 7.083 & 9.250 & 23.000 & ${ }^{*} 90.375$ \\
\hline & $\bar{B}-\mathrm{Y}$ & 11.000 & 6.667 & 9.417 & 31.833 & *65.700 & $* * 150.000$ \\
\hline \multirow[t]{3}{*}{ Left } & Lum & 8.250 & 7.667 & 6.917 & 11.167 & 37.083 & $* 49.000$ \\
\hline & $\overline{R-G}$ & 7.167 & 7.917 & 7.167 & 16.083 & 37.500 & $* 100.750$ \\
\hline & $\overline{B-Y}$ & 16.000 & 12.417 & 18.500 & $* 45.500$ & $* 86.500$ & $* * 150.000$ \\
\hline \multirow[t]{3}{*}{ Right } & Lum & 8.583 & 8.083 & 7.583 & 9.167 & 42.583 & $* 85.750$ \\
\hline & R-G & 7.250 & 7.750 & 6.333 & 13.833 & 35.417 & $* 103.500$ \\
\hline & $\overline{B-Y}$ & 16.417 & 13.750 & 19.750 & $* 47.750$ & *83.000 & ${ }^{*} 114.000$ \\
\hline
\end{tabular}

Table 5: Mean Detection Thresholds in $x y Y$ Space. $Y=5 \mathrm{~cd} / \mathrm{m}^{2}$. Representative chromaticity is white with chromaticity coordinates $(0.33,0.35)$.

Consider the transitions with a horizontal orientation and a spatial frequency of $0.5 \mathrm{cy}$ cles/degree. The luminance transition has a mean detection threshold of 8.167 as seen in Table 5. The $\left(\begin{array}{lll}X_{0} & Y_{0} & Z_{0}\end{array}\right)^{T}$ value for the white representative chromaticity at a luminance value of $Y=5 \mathrm{~cd} / \mathrm{m}^{2}$ is $(4.7145 .0004 .571)^{T}$. Using Eq. (22) gives the $X Y Z$ tristimulus values of $(4.8105 .1014 .664)^{T}$ and $(4.6194 .8994 .479)^{T}$. Converting these vectors to $L^{*} a^{*} b^{*}$ space gives the vectors $\left(\begin{array}{llll}43.733 & 1.7662 .852\end{array}\right)^{T}$ and $(42.9321 .7432 .814)^{T}$. Taking the Euclidean norm of the difference of these two vectors gives a mean detection threshold value of 0.803 .

Since the two chrominance components are calculated in the same manner, we shall show how the mean detection threshold for the red-green direction is computed. The $x y Y$ threshold value is 4.667 for the horizontal orientation and spatial frequency of 0.5 cycles/degree. The two vectors in this color space are found by using Eq. (19). They are $\left(\begin{array}{llll}0.333 & 0.348 & 0.319\end{array}\right)^{T}$ and 
$(0.3270 .3520 .321)^{T}$. Using Eq. $(21)$, one gets the $X Y Z$ tristimulus values of $(4.7815 .0004 .573)^{T}$ and $(4.6495 .0004 .5705)^{T}$. Transforming these vectors to $L^{*} a^{*} b^{*}$ space yields the vectors $\left(\begin{array}{lll}43.335 & 2.9582 .819\end{array}\right)^{T}$ and $\left(\begin{array}{lll}43.335 & 0.5512 .847\end{array}\right)^{T}$. Notice that since the transition was only in chrominance, the $L^{*}$ values are the same. The Euclidean norm of the difference gives a mean detection value of 2.407. Continuing this process with the other spatial frequencies and spatial directions gives Table 6 .

\begin{tabular}{|l|l|r|r|r|r|r|r|}
\hline Spatial & Color & \multicolumn{6}{|c|}{ Spatial Frequency cycles/deg } \\
\cline { 2 - 8 } Dir. & Dir. & 0.5 & 1.0 & 2.0 & 5.0 & 10.0 & 20.0 \\
\hline \multirow{3}{*}{ Hor. } & Lum & 0.803 & 0.663 & 0.622 & 0.712 & 1.327 & 6.426 \\
\cline { 2 - 8 } & R-G & 2.407 & 2.450 & 2.450 & 3.697 & 8.986 & 40.093 \\
\cline { 2 - 8 } & B-Y & 4.471 & 2.299 & 2.618 & 12.544 & 27.169 & 60.447 \\
\hline Vert. & Lum & 0.688 & 0.671 & 0.614 & 0.671 & 2.212 & 7.698 \\
\cline { 2 - 8 } & R-G & 2.622 & 2.880 & 3.654 & 4.771 & 11.868 & 46.873 \\
\cline { 2 - 8 } & B-Y & 4.216 & 2.555 & 3.609 & 12.223 & 25.406 & 60.447 \\
\hline Left & Lum & 0.811 & 0.753 & 0.680 & 1.097 & 3.649 & 4.828 \\
\cline { 2 - 8 } & R-G & 3.697 & 4.084 & 3.697 & 8.297 & 19.361 & 52.325 \\
\cline { 2 - 8 } & B-Y & 6.134 & 4.759 & 7.093 & 17.510 & 33.681 & 60.447 \\
\hline Right & Lum & 0.843 & 0.794 & 0.745 & 0.901 & 4.193 & 8.498 \\
\cline { 2 - 8 } & R-G & 3.740 & 3.998 & 3.267 & 7.136 & 18.283 & 53.774 \\
\cline { 2 - 8 } & B-Y & 6.294 & 5.270 & 7.573 & 18.384 & 32.277 & 44.939 \\
\hline
\end{tabular}

Table 6: Mean Detection Thresholds in $L^{*} a^{*} b^{*}$ Space. $Y=5 c d / m^{2}$. Representative chromaticity is white with chromaticity coordinates $(0.33,0.35)$.

\section{4-4 Calculation of Perceptual Weights}

Once one has obtained the mean detection thresholds in the desired color space, the weights for the given subband configuration must be calculated. The visual experiments provide data at $0.5,1.0$, 2.0, 5.0, 10.0 and 20.0 cycles/degree. The experiment was designed with the subjects at seven feet from the display so that the images subtended two degrees at the eye. The mean detection threshold data must be converted from cycles/degree to cycles/inch for some standard viewing distance. A $256 \times 256$ pixel image has physical dimensions of $3 \times 3$ inches on the monitor. This corresponds to a sample spacing $T_{a}=0.01172$ inches/pixel in both the vertical and horizontal directions, or a sampling frequency $F_{s}=1 / T_{s}=85.33 \mathrm{cycles} /$ inch. Assuming that there was no aliasing in the original digital image implies that the image is bandlimited to a single-sided bandwidth of 42.66 cycles/inch in each direction.

A schematic of the experimental set-up is shown in Figure 6. Define the visual axis as the 
line that is perpendicular to the image and runs through its center. For a given viewing distance, let $\theta$ represent the angle between the point on the visual axis at this distance and the edge of the image. Twice this angle is the angle subtended at the eye by the entire image. Assuming that $\tan (\theta)=\theta$ allows one to convert the data to cycles/inch. For example, consider a viewing distance of five times the picture height. $\theta=5.711$ degrees, and this angle covers 1.5 inches of the image. Therefore, one degree equals 0.2627 inches.

\section{Experiment Geometry}

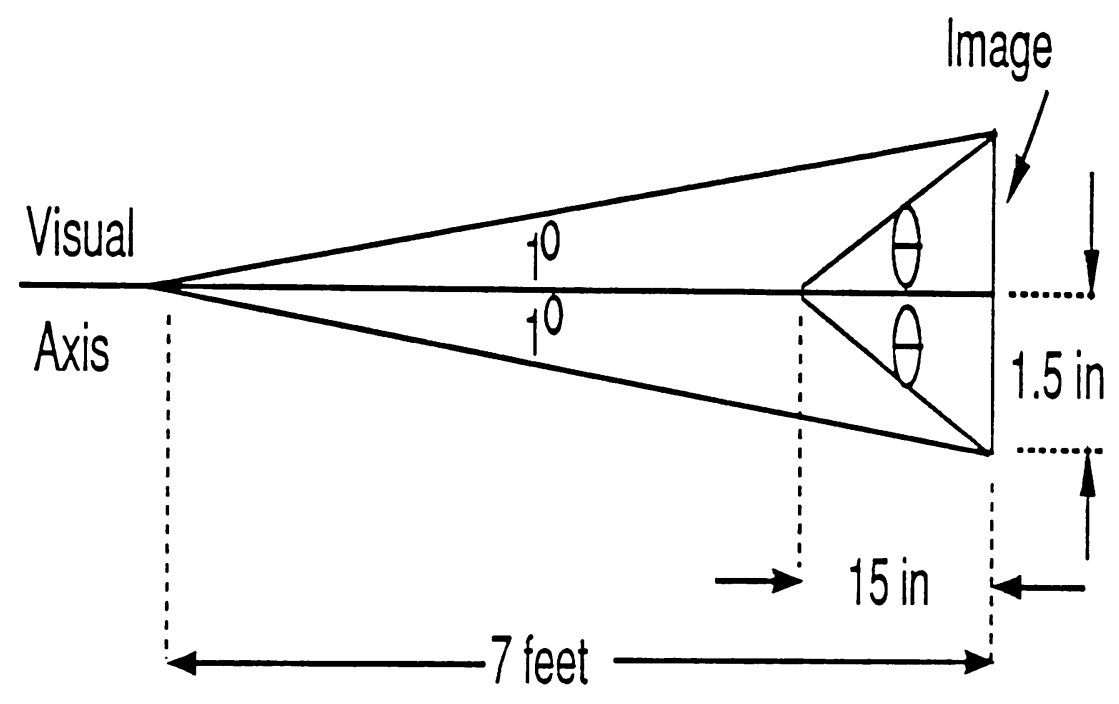

Figure 6: Schematic of the Mean Detection Threshold Experiment.

The perceptual weights are defined to be the mean detection thresholds at the desired spatial frequencies, orientations, and color transition directions. The data for the white background at a luminance value of $Y=5 \mathrm{~cd} / \mathrm{m}^{2}$ is used. The white background was chosen since without any $a$ priori knowledge of the input, one can not assume that the average background color is red, green, or blue. A neutral color such as white is a better assumption. The luminance value was chosen to be $5 \mathrm{~cd} / \mathrm{m}^{2}$ since the histogram of the toy store image has a peak at the lower luminance levels. The choice of background luminance does not have a great effect on the bit allocation.

The use of perceptual weights is an attempt to correct for the non-uniform response of the HVS. Since the mean detection threshold is the smallest change along a color direction that can be detected, it is reasonable to equate the perceptual weights with this threshold. Higher values imply a decreased sensitivity of the human visual system. In Eq. (14), the perceptual weights are 
in the denominator. This means that the higher the value of a weight, the more the distortion for that subband is reduced. Since the total distortion is to be minimized, this implies that fewer bits will be allocated to a subband if its perceptual weight is high. This corresponds to the subband not being perceptually important.

\section{4-4.1 Rectangular Subband Configuration}

Table 7 contains the center frequencies of the subbands measured radially from the origin and shows their orientations. These frequencies will be the ones used to determine the perceptual weights, and they are shown in Figure 7. These center frequencies were converted to cycles/degree for the given viewing distance, five picture heights, and linear interpolation in the desired color space was used to obtain the mean threshold values. Since the lowest frequency subimage contains the baseband information, an average of the horizontal and vertical orientation data was used. These two orientations provided very similar thresholds for this spatial frequency, justifying the use of the average. By using this average of horizontal and vertical orientations instead of a diagonal orientation at the vector sum of them, the lowest frequency subband is given slightly more importance. Even though subbands 122 and 22 are rectangular in shape, they contain the diagonal high-frequency information. These two subbands do not make a distinction between the left and right diagonal orientations so an average of the two was used.

The set of perceptual weights in $L^{*} a^{*} b^{*}$ space, based on a viewing distance of five times the picture height, is given in Table 8. Since the blue-yellow direction is not a straight line in $L^{*} a^{*} b^{*}$ space, it was approximated by a straight line at an angle of $-59.62^{\circ}$ from the $a^{*}$ axis. The weights for the transitions in the $b^{*}$ direction were obtained by assuming the blue-yellow weights lie on this line, and projecting this line onto the $b^{*}$ axis. The result is that these weights were multiplied by $\cos \left(30.38^{\circ}\right)$.

The set of perceptual weights in $A C_{1} C_{2}$ space is given in Table 9, while Table 10 contains the set of weights in $Y I^{*} Q^{*}$ space. The weights for the $Q^{*}$ direction were obtained by projecting the weights for the blue-yellow direction in $Y I^{*} Q^{*}$ space onto the $Q^{*}$ axis. Since the angle between the blue-yellow direction and the $Q^{*}$ axis was only $14.4^{\circ}$, the effect of this change was negligible, but it was done to be consistent with processing in $L^{*} a^{*} b^{*}$ space.

These sets of weighting factors have been used to obtain simulation results for all three cases. To apply these weighting factors to Case 1 , a single value is needed for the higher subbands. We 


\begin{tabular}{|c|c|c|c|}
\hline Subband & $\begin{array}{c}\text { Center Freq. } \\
\text { cycles/inch }\end{array}$ & $\begin{array}{c}\text { Center Freq. } \\
\text { cycles/deg. }\end{array}$ & Orientation \\
\hline 111 & 5.333 & 1.401 & $1 / 2 \mathrm{H}, 1 / 2 \mathrm{~V}$ \\
112 & 16.000 & 4.203 & $\mathrm{~V}$ \\
121 & 16.000 & 4.203 & $\mathrm{H}$ \\
122 & 22.627 & 5.944 & $1 / 2 \mathrm{~L}, 1 / 2 \mathrm{R}$ \\
\hline 12 & 32.000 & 8.406 & $\mathrm{~V}$ \\
21 & 32.000 & 8.406 & $\mathrm{H}$ \\
22 & 45.255 & 11.888 & $1 / 2 \mathrm{~L}, 1 / 2 \mathrm{R}$ \\
\hline
\end{tabular}

Table 7: Subband Orientation. Center frequencies in cycles/degree are for a viewing distance of 5 picture heights.

Seven Subband System

Rectangular Configuration

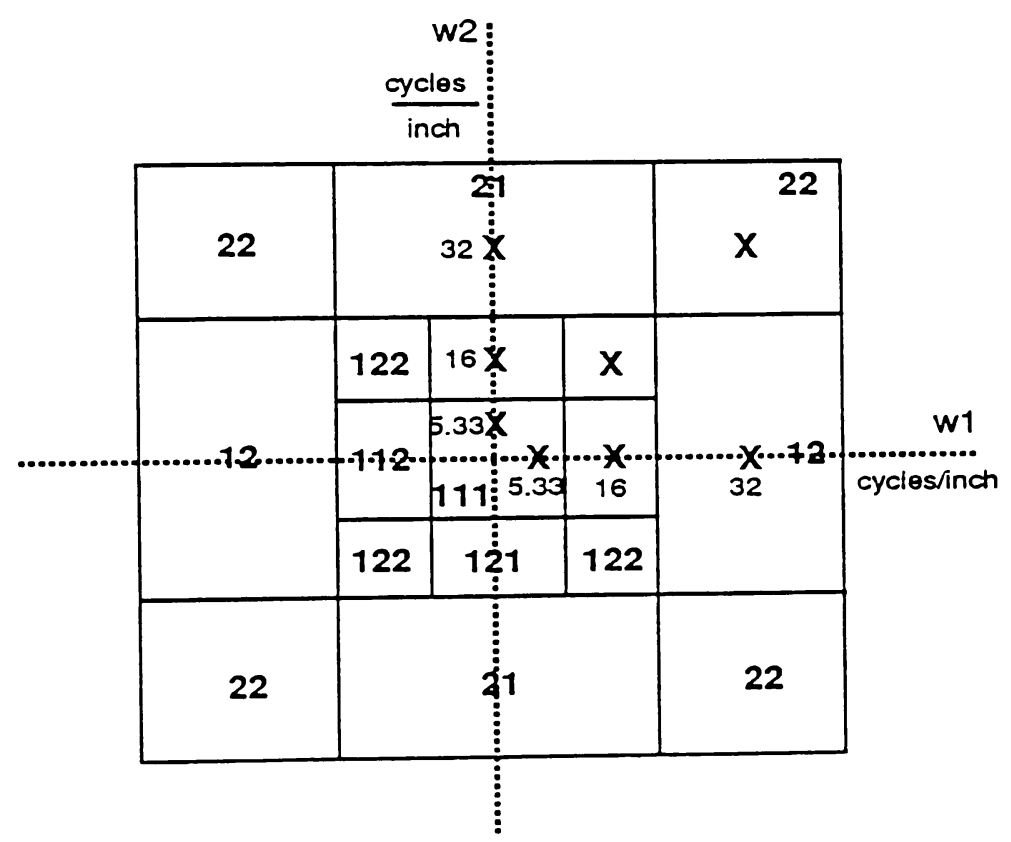

Figure 7: Spatial Frequency Locations of the Perceptual Weights. The bold numerals are the numbers of the subbands. The smaller numerals are the spatial frequencies in cycles/inch.

\begin{tabular}{|c|ccc|}
\hline Subband & \multicolumn{3}{|c|}{ Weights } \\
& $L^{*}$ & $a^{*}$ & $b^{*}$ \\
\hline 111 & 0.648 & 2.820 & 2.331 \\
112 & 0.656 & 4.474 & 8.570 \\
121 & 0.689 & 3.366 & 8.547 \\
122 & 1.551 & 9.813 & 17.931 \\
\hline 12 & 1.721 & 9.605 & 18.292 \\
21 & 1.131 & 7.300 & 19.417 \\
22 & 4.439 & 25.284 & 31.662 \\
\hline
\end{tabular}

Table 8: $L^{*} a^{*} b^{*}$ Perceptual Weights. 5 Times Picture Height. White point is $(34.5137 .3637 .16)^{T}$. 


\begin{tabular}{|c|ccc|}
\hline Subband & \multicolumn{3}{|c|}{ Weights } \\
& $A$ & $C_{1}$ & $C_{2}$ \\
\hline 111 & 0.739 & 1.391 & 0.694 \\
112 & 0.749 & 2.207 & 2.552 \\
121 & 0.786 & 1.660 & 2.545 \\
122 & 1.771 & 4.841 & 5.343 \\
\hline 12 & 1.964 & 4.738 & 5.450 \\
21 & 1.290 & 3.600 & 5.786 \\
22 & 5.077 & 12.505 & 9.452 \\
\hline
\end{tabular}

Table 9: $A C_{1} C_{2}$ Perceptual Weights. 5 Times Picture Height.

\begin{tabular}{|c|ccc|}
\hline Subband & \multicolumn{3}{|c|}{ Weights } \\
& $Y$ & $I^{*}$ & $Q^{*}$ \\
\hline 111 & 0.164 & 0.274 & 0.175 \\
112 & 0.166 & 0.434 & 0.644 \\
121 & 0.174 & 0.327 & 0.643 \\
122 & 0.392 & 0.952 & 1.355 \\
\hline 12 & 0.435 & 0.932 & 1.382 \\
21 & 0.286 & 0.708 & 1.468 \\
22 & 1.120 & 2.457 & 2.421 \\
\hline
\end{tabular}

Table 10: $Y I^{*} Q^{*}$ Perceptual Weights - 5 Times Picture Height.

have used the luminance component for the weight of each of these subbands since the luminance is more important. This gives slightly too little weight to the chrominance components of the lowest frequency subband, but the results are visually very good. Further discussion of this point will occur in the section on experimental results.

\section{Experimental Results}

The results of the computer simulations presented in this section attempt to answer three questions. The first one is how much improvement in reconstructed image quality can be obtained by the use of a perceptually optimal bit allocation. The use of perceptual weights in the Marginal Analysis bit allocation method is compared to the use of uniform weights. For conciseness, we refer to these as the perceptual method and the uniform method, respectively. The second question is to determine how well the three different vector quantizer cases compare for a number of different compression ratios. To answer this, the results will emphasize coding in $L^{*} a^{*} b^{*}$ space. The third question addresses the advantages and disadvantages of coding in the three different color spaces.

The first set of experiments examine the differences between coding with the uniform weights 


\begin{tabular}{|c||c|c|c||c|c|c|}
\hline \multicolumn{1}{|c||}{} & \multicolumn{3}{c||}{ Case 1 } & \multicolumn{3}{c|}{ Case 2 } \\
\hline Subband & $\begin{array}{c}Y I^{*} Q^{*} \\
\text { bits/pix }\end{array}$ & $\begin{array}{c}L^{*} a^{*} b^{*} \\
\text { bits/pix }\end{array}$ & $\begin{array}{c}A C_{1} C_{2} \\
\text { bits/pix }\end{array}$ & $\begin{array}{c}Y I^{*} Q^{*} \\
\text { bits/pix }\end{array}$ & $\begin{array}{c}L^{*} a^{*} b^{*} \\
\text { bits/pix }\end{array}$ & $\begin{array}{c}A C_{1} C_{2} \\
\text { bits/pix }\end{array}$ \\
\hline 111 & $7,6,6$ & $6,5,5$ & $6,5,4$ & $7,6,6$ & $6,5,6$ & $7,6,4$ \\
112 & 6 & 5 & 5 & $9,9,5$ & $5,8,4$ & $9,9,3$ \\
121 & 6 & 3 & 4 & $9,0,0$ & $6,5,0$ & $8,3,0$ \\
122 & 1 & 0 & 0 & $0,0,0$ & $0,0,0$ & $0,0,0$ \\
\hline 12 & 0 & 2 & 2 & $0,5,0$ & $0,6,2$ & $0,7,0$ \\
21 & 0 & 0 & 0 & $0,0,0$ & $0,0,0$ & $0,0,0$ \\
22 & 0 & 0 & 0 & $0,0,0$ & $0,0,0$ & $0,0,0$ \\
\hline
\end{tabular}

Table 11: Bit Allocation - Uniform Weighting. 12:1 Compression Ratio.

and the perceptual weights. At compression ratios of $4: 1$ and $8: 1$, the visual differences between the two methods is very small. Both methods provide enough bits to the lowest frequency subband; the allocation of enough bits to this subband is essential for high quality reproduction. The luminance component receives at least six bits/pixel, and this is enough to prevent contouring. The chrominance components each receive at least five bits/pixel. At a compression ratio of 8:1, the differences in bit allocations between the two weighting methods is apparent for Case 1 . In $L^{*} a^{*} b^{*}$ space, the uniform weighting gives the lowest frequency luminance component six bits/pixel compared to seven for the perceptual weighting. There is a slight bit of contouring in the GIRL image for the former method. Both methods yield high quality results for Case 2.

The differences between the two methods can be more easily seen at compression ratios of 12:1 and higher. For this compression ratio, the uniform weighting bit allocations are given in Table 11, and the corresponding bit allocations using perceptual weighting are in Table 12 . The Case 1 allocations for the two methods are similar. The perceptual method adds slightly more bits to the lower frequency subbands at the expense of the higher frequency subbands. Because of the extra degrees of freedom in Case 2, the bit allocations are more different. By reducing the number of bits given to the three highest frequency subbands, the perceptual method can allocate more bits to all three color components of the four lower frequency subbands.

A close examination of the Case 2 images at a compression ratio of 12:1 shows a noticeable improvement in the perceptually weighted images. This is especially true for the GIRL image, where the mottled appearance of the forehead is significantly smoothed. Table 13 contains the average $\Delta E$ errors for the uniform method and Table 14 contains the errors for the perceptual method. While the use of this statistic does not correlate very well with the subjective quality, it still shows that the errors using the perceptual method are less. These differences are accentuated 


\begin{tabular}{|c||c|c|c||c|c|c|}
\hline \multicolumn{1}{|c||}{} & \multicolumn{3}{c||}{ Case 1 } & \multicolumn{3}{c|}{ Case 2 } \\
\hline Subband & $\begin{array}{c}Y I^{*} Q^{*} \\
\text { bits/pix }\end{array}$ & $\begin{array}{c}L^{*} a^{*} b^{*} \\
\text { bits/pix }\end{array}$ & $\begin{array}{c}A C_{1} C_{2} \\
\text { bits/pix }\end{array}$ & $\begin{array}{c}Y I^{*} Q^{*} \\
\text { bits/pix }\end{array}$ & $\begin{array}{c}L^{*} a^{*} b^{*} \\
\text { bits/pix }\end{array}$ & $\begin{array}{c}A C_{1} C_{2} \\
\text { bits/pix }\end{array}$ \\
\hline 111 & $7,6,6$ & $6,5,5$ & $7,5,5$ & $8,6,7$ & $7,6,6$ & $7,6,5$ \\
112 & 7 & 7 & 7 & $9,7,3$ & $9,9,3$ & $9,9,3$ \\
121 & 6 & 5 & 4 & $9,8,0$ & $9,6,0$ & $9,2,0$ \\
122 & 0 & 0 & 0 & $0,0,0$ & $0,0,0$ & $0,0,0$ \\
\hline 12 & 0 & 1 & 1 & $0,2,0$ & $0,4,0$ & $0,6,0$ \\
21 & 0 & 0 & 0 & $0,0,0$ & $0,0,0$ & $0,0,0$ \\
22 & 0 & 0 & 0 & $0,0,0$ & $0,0,0$ & $0,0,0$ \\
\hline
\end{tabular}

Table 12: Bit Allocation - Perceptual Weighting. 12:1 Compression Ratio. 5 Times Picture Height.

\begin{tabular}{|c||r|r|r|r|}
\hline Color Space & Case & Image & $\Delta \mathrm{E}$ (ave) & $\sigma_{\Delta E}$ \\
\hline$Y I^{*} Q^{*}$ & 1 & GIRL & 4.083 & 4.169 \\
& 1 & DOLL & 4.412 & 3.964 \\
\hline$L^{*} a^{*} b^{*}$ & 1 & GIRL & 2.920 & 2.448 \\
& 1 & DOLL & 3.022 & 2.159 \\
\hline$A C_{1} C_{2}$ & 1 & GIRL & 3.518 & 2.428 \\
& 1 & DOLL & 3.798 & 2.580 \\
\hline \hline$Y I^{*} Q^{*}$ & 2 & GIRL & 3.319 & 2.957 \\
& 2 & DOLL & 3.783 & 3.285 \\
\hline$L^{*} a^{*} b^{*}$ & 2 & GIRL & 2.450 & 1.776 \\
& 2 & DOLL & 2.723 & 1.860 \\
\hline$A C_{1} C_{2}$ & 2 & GIRL & 3.052 & 2.002 \\
& 2 & DOLL & 3.488 & 2.361 \\
\hline
\end{tabular}

Table 13: Distortion - Uniform Weighting. 12:1 Compression Ratio.

at a compression ratio of $16: 1$. Figures 10 and 11 , at the end of this paper, show the reconstructed GIRL and DOLL images after coding in $L^{*} a^{*} b^{*}$ space. Each figure contains photographs of the images resulting from the two different methods of bit allocation. The uniform method images are clearly degraded, while the perceptual ones do not look much different for those at compression ratios of $8: 1$ and 12:1. There is a slight loss of high frequency detail noticeable when compared to the original.

The second set of experiments examine the three cases to determine the range of compression ratios where they yield good quality images. Perceptual weighting was used in all three cases. Figures 8 and 9 show the plots of the average $\Delta E$ distortion as a function of compression ratio for the GIRL and DOLL images coded in $L^{*} a^{*} b^{*}$ space, respectively. One can see that the image quality slowly degrades as the compression ratio is increased. This change in quality is fairly constant over a wide range of compression ratios. Cases 1 and 2 yield similar quality images for 


\begin{tabular}{|c||r|r|r|r|}
\hline Color Space & Case & Image & $\Delta \mathrm{E}$ (ave) & $\sigma_{\Delta E}$ \\
\hline$Y I^{*} Q^{*}$ & 1 & GIRL & 3.820 & 3.651 \\
& 1 & DOLL & 4.190 & 3.599 \\
\hline$L^{*} a^{*} b^{*}$ & 1 & GLRL & 3.206 & 2.720 \\
& 1 & DOLL & 3.239 & 2.469 \\
\hline$A C_{1} C_{2}$ & 1 & GIRL & 4.183 & 2.806 \\
& 1 & DOLL & 4.083 & 2.816 \\
\hline \hline$Y I^{*} Q^{*}$ & 2 & GIRL & 3.579 & 3.440 \\
& 2 & DOLL & 4.188 & 3.747 \\
\hline$L^{*} a^{*} b^{*}$ & 2 & GLRL & 2.633 & 2.147 \\
& 2 & DOLL & 2.857 & 2.042 \\
\hline$A C_{1} C_{2}$ & 2 & GIRL & 2.840 & 1.974 \\
& 2 & DOLL & 3.276 & 2.284 \\
\hline
\end{tabular}

Table 14: Distortion - Perceptual Weighting. 12:1 Compression Ratio. 5 Times Picture Height.

the lower compression ratios, but Case 2 provides better results at and above a compression ratio of 12:1. At still higher compression ratios, i.e. 20:1 and greater, almost all of the bits are allocated to the lowest frequency subband. Cases 1 and 2 become the same, since both cases scalar quantize the lowest frequency subband.

In Case 3, the chrominance components of the lowest frequency subband are vector quantized. The maximum number of bits allowed for the chrominance components of this subband is set at 12. This is done for two reasons. The first is to limit the computational complexity of the coding system. The second reason was that at 11 and 12 bits/vector, the LBG algorithm did not use the entire number of codevectors allowed. This is mainly due to the size of the training sequence, and to the value of the perturbation used in the splitting. Therefore, Case 3 was only run for compression ratios of 16:1 and greater. Even at 16:1, the bit allocation algorithm gave all 12 bits/vector to each chrominance component. For the GIRL image, Figure 8 shows that Case 3 provides better results than Case 2. This was most visible at a compression ratio of 20:1. The DOLL image contains many saturated colors. Limiting the number of bits/vector lessened the effectiveness of Case 3 for this image. Even so, the results were still slightly better than for Case 2.

The last question to be answered is which color space should be used for processing. $Y I Q$ packs most of the energy in the luminance component. The perceptual weights ensure that sufficient bits are given to this component to eliminate contouring. In this respect, this color space is better than the other two. However, for highly saturated colors, the color errors are higher in this space. This is seen both in the higher average $\Delta E$ errors for this space, and by examining the reconstructed images. Both $L^{*} a^{*} b^{*}$ and $A C_{1} C_{2}$ space provide better color fidelity. 


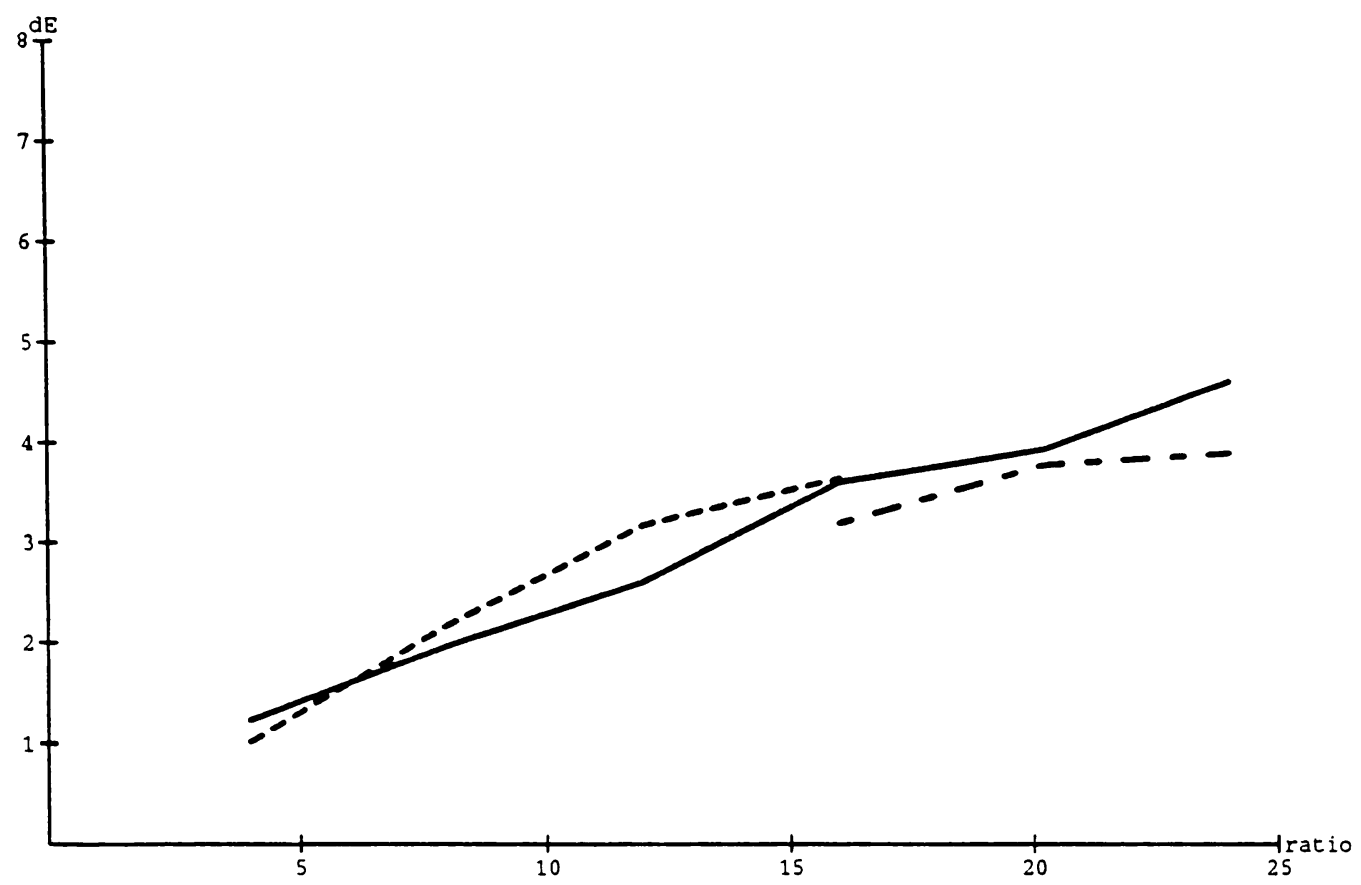

Figure 8: Average $\Delta E$ Distortion of the GIRL Image in $L^{*} a^{*} b^{*}$ Space versus Compression Ratio. The dotted line is Case 1 , the solid line is Case 2, and the dot-dashed line is Case 3.

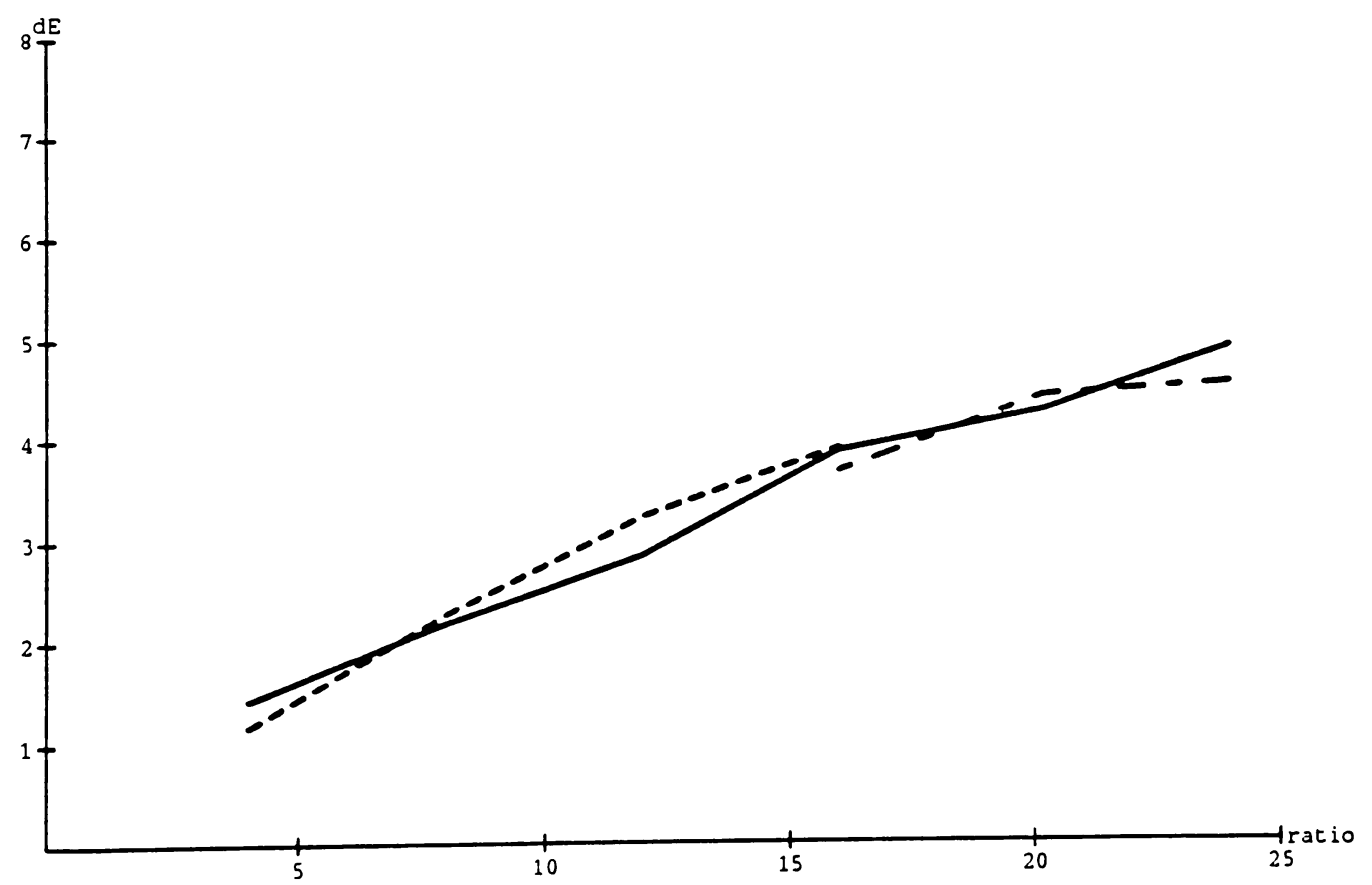

Figure 9: Average $\Delta E$ Distortion of the DOLL Image in $L^{*} a^{*} b^{*}$ Space versus Compression Ratio. The dotted line is Case 1 , the solid line is Case 2, and the dot-dashed line is Case 3. 


\section{Conclusions}

The combination of subband coding with vector quantization can yield images of very high quality while providing compression ratios exceeding 10:1. The use of a perceptually optimal bit allocation algorithm is integral to achieving these results at the higher compression ratios. Vector quantizing the color components separately adds extra degrees of freedom and leads to an increased performance. For compression ratios of $20: 1$ and greater, the chrominance components of the lowest frequency component should also be vector quantized. Further improvements can be obtained by increasing the size of the vectors used in the higher frequency subbands.

The use of a perceptually uniform color space yields better color reproduction, but can also lead to larger color artifacts in localized areas. For moderate compression ratios, the color artifacts are small enough that coding should be done in a perceptually uniform color space. Of the two examined, $L^{*} a^{*} b^{*}$ space has fewer areas of large color errors than $A C_{1} C_{2}$ space. The latter space has an exponential in the inverse transformation; color errors caused by the quantization are enhanced more in this space than in the former. If some error in the average colors of objects can be tolerated, $Y I Q$ space provides reconstructed images containing little contouring.

\section{References}

[1] M. Antonini, M. Barlaud, P. Mathieu, and I. Daubechies, "Image Coding Using Vector Quantization in the Wavelet Transform Domain," Proc. ICASSP, pp. 2297-2300, 1990.

[2] C.K. Chan and J.C. Chow, "Optimal Display of True Color Images Using Vector Quantization Techniques," Proc. IEEE VSPC, pp. 5-8, 1991.

[3] H. Everett III, "Generalized Lagrange Multiplier Method for Solving Problems of Optimum Allocation of Resources," Operations Research, vol. 11, pp. 399-417, 1963.

[4] O.D. Faugeras, "Digital Color Image Processing Within the Framework of a Human Visual Model," IEEE. Trans. on ASSP., vol. ASSP-27, pp. 380-393, Aug. 1979.

[5] O.D. Faugeras, "Digital Color Image Processing and Psychophysics Within the Framework of a Human Visual Model," Doctoral Dissertation, Dept. of Computer Science, University of Utah, Jun. 1976.

[6] T.R. Fischer, "On the Rate-Distortion Efficiency of Subband Coding," Proc. Globecom, pp. 3.6.1-3.6.3, 1991.

[7] B. Fox, "Discrete Optimization via Marginal Analysis," Management Science, vol. 13, pp. 210-216, Nov. 1966.

[8] J.D. Johnston, "A Filter Family Designed for use in Quadrature Mirror Filter Banks", Proc. ICASSP, pp. 291-294, Apr. 1980. 
[9] C. Kim, J. Bruder, M. Smith, and R. Mersereau, "Subband Coding of Color Images Using Finite State Vector Quantization," Proc. ICASSP, pp. 753-756, 1988.

[10] B. Krishnakumar, "Visual Sensitivity to Color-Varying Stimuli," Master's thesis, Department of Electrical and Computer Engineering, North Carolina State University, Dec. 1991.

[11] Y. Linde, A. Buzo, and R.M. Gray, "An Algorithm for Vector Quantizer Design," IEEE Trans. on Comm., vol. Com-28, pp. 84-95, Jan. 1980.

[12] D.L. MacAdam, "Uniform Color Scales," Journal of the Optical Society of America, vol. 64, pp. 1691-1702, Dec. 1974.

[13] V. Mani, "Calibration of Color Monitors," Master's thesis, Department of Electrical and Computer Engineering, North Carolina State University, 1991.

[14] S.A. Rajala, H.J. Trussell, and B. Krishnakumar, "Visual Sensitivity to Color-Varying Stimuli," SPIE/ISET's Symposium on Electronic Imaging Science \& Technology, Feb. 1992.

[15] A. Segall, "Bit Allocation and Encoding of Vector Sources," IEEE Trans. on Info. Theory, vol IT-22, pp. 162-169, Mar. 1976.

[16] Y. Shoham and A. Gersho, "Efficient Bit Allocation for an Arbitrary Set of Quantizers," IEEE Trans. on $A S S P$, vol. ASSP-36, pp. 1445-1453, Sep. 1988.

[17] W.N. Sproson, Colour Science in Television and Display Systems, Adam Hilger Ltd., Bristol, 1983.

[18] A.V. Trushkin, "Bit Number Distribution Upon Quantization of a Multivariate Random Variable," Problems of Information Transmission, vol. 16, pp. 76-79, 1980.

[19] A.V. Trushkin, "Optimal Bit Allocation Algorithm for Quantizing a Random Vector," Problems of Information Transmission, vol. 17, pp. 156-161, 1981.

[20] R.E. Van Dyck and S.A. Rajala, "Sensitivity to Color Errors Introduced by Processing in Different Color Spaces", Proc. IEEE VSPC, pp. 192-195, 1991.

[21] R.E. Van Dyck and S.A. Rajala, "Design of Quantizers for Alternative Color Spaces," Presented at IEEE MDSP Workshop, p. 7.4, 1991.

[22] R.E. Van Dyck and S.A. Rajala, "Subband/VQ Coding in Perceptually Uniform Color Spaces," Proc. ICASSP, pp. 237-240, 1992.

[23] P.H. Westerink, J. Biemond, and D.E. Boekee, "An Optimal Bit Allocation Algorithm for Sub-Band Coding," Proc. ICASSP, pp. 757-760, 1988.

[24] P.H. Westerink, D.E. Boekee, J. Biemond, and J.W. Woods, "Subband Coding of Images Using Vector Quantization," IEEE Trans. on Comm., vol. Com-36, pp. 713-719, 1988.

[25] G. Wyszecki and W.S. Stiles, Color Science: Concepts and Methods, Quantitative Data and Formulae, John Wiley \& Sons, New York, pp. 164-169. 1982. 

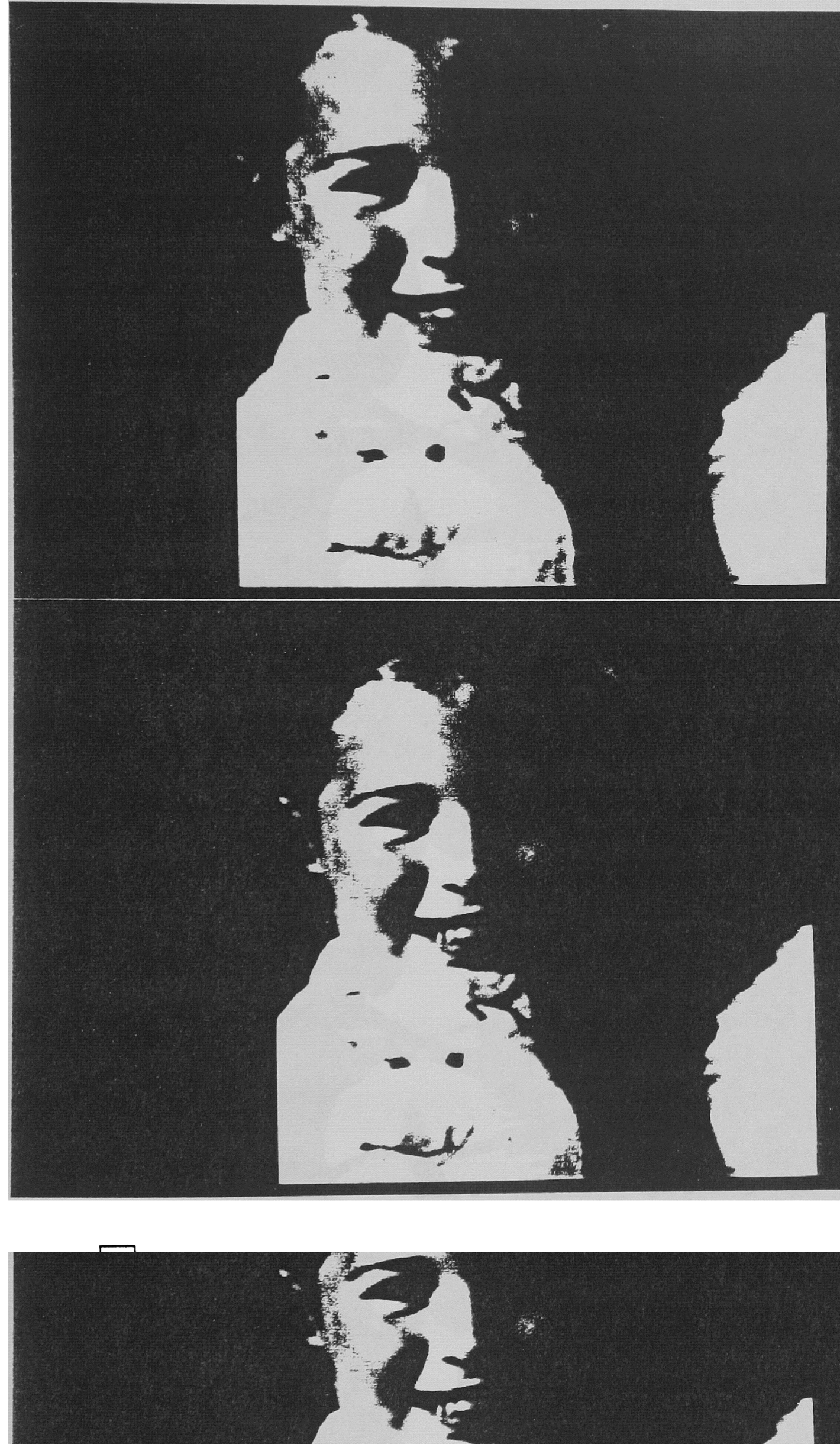


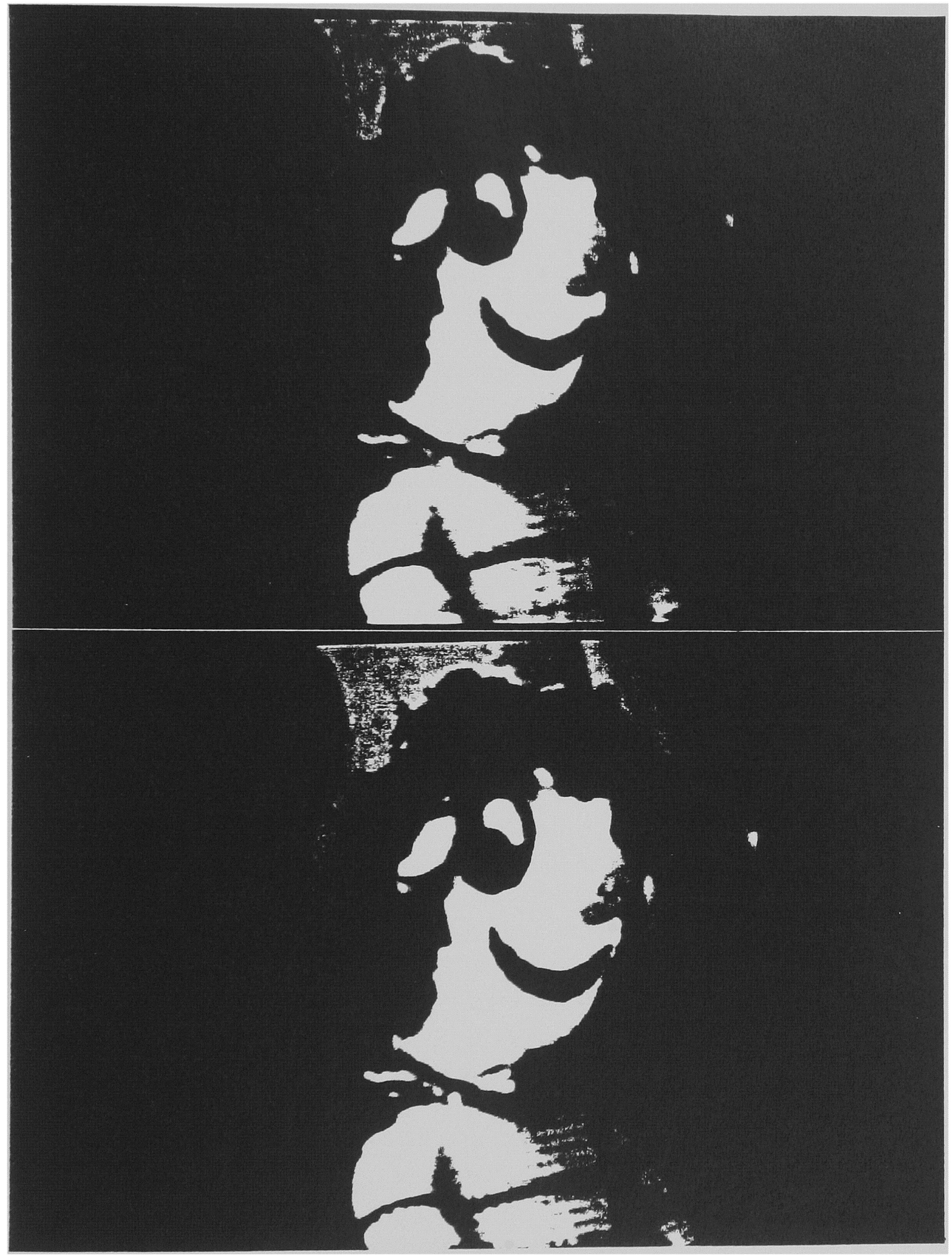

